OPEN ACCESS

Edited by:

Jian Cheng,

Beihang University, China

Reviewed by:

Xiran Jiang,

China Medical University, China Kuang Gong

Harvard Medical School,

United States

*Correspondence:

Dinggang Shen

dinggang.shen@gmail.com

†These authors have contributed equally to this work and share first authorship

Specialty section:

This article was submitted to Artificial Intelligence in Radiology,

a section of the journal

Frontiers in Radiology

Received: 23 September 2021 Accepted: 08 November 2021 Published: 13 December 2021

Citation:

Wang S, Cao G, Wang Y, Liao S,

Wang Q, Shi J, Li C and Shen D (2021) Review and Prospect: Artificial

Intelligence in Advanced Medical

Imaging. Front. Radiol. 1:781868.

doi: 10.3389/fradi.2021.781868

\section{Review and Prospect: Artificial Intelligence in Advanced Medical Imaging}

\author{
Shanshan Wang ${ }^{1,2+}$, Guohua Cao ${ }^{3 \dagger}$, Yan Wang ${ }^{4 t}$, Shu $\mathrm{Liao}^{5 t}$, Qian Wang ${ }^{3 t}$, Jun Shi ${ }^{6 \dagger}$, \\ Cheng $\mathrm{Li}^{1}$ and Dinggang Shen ${ }^{3,5 *}$ \\ ${ }^{1}$ Paul C. Lauterbur Research Center for Biomedical Imaging, Shenzhen Institute of Advanced Technology, Chinese Academy \\ of Sciences (CAS), Shenzhen, China, ${ }^{2}$ Pengcheng Laboratrory, Shenzhen, China, ${ }^{3}$ School of Biomedical Engineering, \\ ShanghaiTech University, Shanghai, China, ${ }^{4}$ School of Computer Science, Sichuan University, Chengdu, China, ${ }^{5}$ Shanghai \\ United Imaging Intelligence Co., Ltd., Shanghai, China, ${ }^{6}$ School of Communication and Information Engineering, Shanghai \\ University, Shanghai, China
}

Artificial intelligence (Al) as an emerging technology is gaining momentum in medical imaging. Recently, deep learning-based Al techniques have been actively investigated in medical imaging, and its potential applications range from data acquisition and image reconstruction to image analysis and understanding. In this review, we focus on the use of deep learning in image reconstruction for advanced medical imaging modalities including magnetic resonance imaging (MRI), computed tomography (CT), and positron emission tomography (PET). Particularly, recent deep learning-based methods for image reconstruction will be emphasized, in accordance with their methodology designs and performances in handling volumetric imaging data. It is expected that this review can help relevant researchers understand how to adapt Al for medical imaging and which advantages can be achieved with the assistance of Al.

Keywords: deep learning, magnetic resonance imaging, computed tomography, positron emission tomography, medical imaging reconstruction

\section{INTRODUCTION}

Of all the advances in modern medicine, medical imaging is among the most remarkable developments. It allows us to see anatomical structures, organs, and biological processes unreachable by unaided eyes, providing tremendous opportunities for scientific research as well as disease diagnosis and treatment $(1,2)$. Different modalities such as magnetic resonance imaging (MRI) (3), computational tomography (CT) (4), and positron emission tomography (PET) (5) can provide versatile information, ranging from structure, morphology to physiological function. Specifically, MRI uses powerful magnetic fields, radio waves, and computers to produce details of anatomical structures and functions $(6,7)$. CT measures the linear attenuation coefficient of tissues inside each voxel element as an X-ray beam transmits through the body. PET measures changes in metabolic processes as well as other physiological activities by counting radioactive emissions of a biochemical metabolite labeled with radioactive material.

To better serve the clinical end-users, abundant studies have been conducted to optimize the scanning process, improve the imaging efficiency, and enhance the image quality of MRI/CT/PET $(8,9)$. Image reconstruction plays a significant role in this aspect. For MRI, its slow imaging speed has been a long-lasting bottleneck that seriously limits its wider applications in the clinic (10). Among different possible solutions, $\mathrm{k}$-space undersampling has been identified as a highly 
effective approach to accelerate the scan $(11,12)$. Nevertheless, images generated from undersampled k-space data are subject to the low-quality issue, with possible loss of the important information related to disease diagnosis or treatment (13). Thus, high-quality image reconstruction from incomplete kspace data is critical. As for CT and PET, the main focus is to reconstruct high-quality images from deteriorated raw data caused by low-dose imaging demands $(14,15)$. Many efforts have been devoted to developing image reconstruction methods for MRI/CT/PET, among which deep learning-based methods have shown unprecedented successes $(9,14-16)$.

During the last decade, deep learning has been extensively applied to medical imaging to handle different problems, such as image reconstruction (17), image registration (18-20), image classification (21, 22), and lesion segmentation (23). Among these applications, image reconstruction is a primary step in the clinical workflow that has a huge impact on the downstream tasks of imaging-based analysis and decision making. Notice that different medical imaging modalities (MRI, CT, and PET) have their own unique imaging physics and principles, and thus numerous deep learning-based methods have been proposed to accomplish respective reconstruction tasks $(9,11,14,15)$. For MRI, existing works have achieved impressive achievement to balance imaging efficiency and imaging quality $(9,11)$. Similarly, promising results have also been achieved for CT and PET image reconstruction $(14,15)$. However, current progress is still preliminary for deep learning-based image reconstruction in real applications, and more efforts are needed to make this technology mature enough for wide real-world clinical applications. Thus, it is the right time to review existing works to help beginners as well as non-specialists better understand this relatively new technique and promote more follow-up investigations and applications.

The remainder of this review paper is organized as follows. In section Overall Workflow of Deep LearningBased Reconstruction, we demonstrate the overall workflow of deep learning-based reconstruction, by briefly introducing the basics of deep learning relevant to the reconstruction task, the purpose of image reconstruction, and the workflow of deep learning-based reconstruction. Detailed technical developments of deep learning-based reconstruction are introduced in section Technical Developments of Deep Learning-Based Reconstruction. Section Clinical Applications and Current Achievements reviews current clinical applications and achievements, followed by descriptions of key challenges and opportunities in section Challenges and Opportunities. Finally, section Conclusion concludes the paper.

\section{OVERALL WORKFLOW OF DEEP LEARNING-BASED RECONSTRUCTION}

\section{Basics of Deep Learning}

Artificial intelligence (AI) refers to the ability of a machine to simulate human intelligence by thinking and acting like humans (24). Deep learning is a sub-discipline of AI, which specifically addresses various tasks through building deep neural networks (DNNs) (25). Different abstract levels of representations are extracted with multi-layer networks which enable the learning of complex functions. When inputs are images, the low-level features usually represent edges and contours in the images, whereas the high-level features are commonly semantic features (26). One key characteristic for deep learning is that all the parameters for feature extraction are learned automatically with the provided data samples, which can be better self-optimized to specific problems compared to the use of manual feature engineering approaches $(26,27)$.

Supervised learning, unsupervised learning, and reinforcement learning are the three major paradigms for deep learning (28-31). Supervised learning requires paired data samples for the inputs and the expected outputs (28). Model optimization is performed by minimizing loss functions that are calculated to measure the difference between model outputs and ground truth. In unsupervised learning, only input data samples are provided, and certain assumptions of the data have to be made and then the corresponding model constraints are enforced to facilitate the model learning (29). In reinforcement learning, an algorithm is referred to as an agent. Then, the agent takes an action to change its state, and, at the same time, a reward or penalty is assigned. Different from supervised learning, the training data of reinforcement learning provide only an indication of whether an action is correct or not. The overall goal of reinforcement learning is to achieve the maximum reward over time by learning a policy for the agent to choose proper actions for any given states (31). Most medical image reconstruction models are based on supervised learning or unsupervised learning, while reinforcement learning is less frequently utilized.

\section{Deep Learning-Based Image Reconstruction \\ MRI}

MRI reconstruction aims to generate high-quality images from sampled k-space data. Conventional reconstruction methods (i.e., Fourier transform) require the scanning process to follow the Nyquist sampling theory. Thus, to obtain highquality images, the sampling frequency should be high enough, which unfortunately makes the scanning process very timeconsuming. On the other hand, undersampling, which breaks the Nyquist sampling theory, leads to imperfect MR image reconstruction if using conventional reconstruction methods. To this end, compressed sensing (CS) MRI (CS-MRI) has been proposed by introducing CS theory to reconstruct MR images with significantly fewer measurements than those required by traditional Nyquist sampling theory (32). CS-MRI accomplishes the reconstruction task mainly by exploiting the sparsity of MRI, since most MR images are sparse after transformed into an appropriate domain (32), such as using total variation (33) and wavelet transformation (34).

Despite the successes achieved, CS-MRI still has limited performance because of using manually-designed methods to exploit the sparsity in MRI. By contrast, deep learning-based image reconstruction for MRI can automatically and fully exploit the available data information and recover the lost information 
under the guidance of certain prior knowledge. Deep learning was first introduced to MR image reconstruction in 2016 by Wang et al. (35). In their work, a three-layer neural network was built to automatically learn the mapping between low-quality and high-quality images (35). Following this work, a series of studies have been published, aiming to build more sophisticated, robust, and optimized deep learning models for MR image reconstruction (36-39).

Existing deep learning-based MR image reconstruction methods can be classified into two major categories, (1) modelbased methods and (2) data-driven methods. Model-based methods reconstruct high-quality MR images via solving certain optimization algorithms and utilizing neural network modules to represent the reconstruction steps of the solution. Typical optimization algorithms include alternating direction method of multipliers (ADMM) algorithm (40), iterative shrinkagethresholding algorithm (ISTA) (41), and primal-dual hybrid gradient (PDHG) algorithm (42). Data-driven methods are the end-to-end approaches that rely on DNNs with large capacities to learn non-linear reconstruction processes. Example models include U-Net (36), residual network (ResNet) (43), and generative adversarial networks (GAN) (44). Model-based methods are more interpretable as the network blocks can correspond to the algorithm solutions, and data-driven methods are more effective in data exploitation. Overall, deep learningbased MR image reconstruction methods have dominated the current research field, with promising performance.

\section{CT}

In $\mathrm{CT}$, image reconstruction aims to transform the sensor data, which basically reflects line integrals of the object, to an image representing the object. Until recently, most CT reconstruction methods can be classified as either analytic reconstruction or iterative reconstruction. Analytic reconstruction is based on the mathematical inverse of the forward model of an imaging process, which could either be mathematically derived or numerically modeled after the design of the CT imaging device and the knowledge about how it generates sensor data. A typical example of analytic reconstruction in CT is filtered back-projection (FBP) (45). Iterative reconstruction is based on a numerical forward model combined with a feedback loop (46-51). In the feedback loop, the error between the calculated sensor dataset and the measured sensor dataset is back-transformed to the image domain to update the current image estimation. This process is repeated until the error reaches a small threshold and the optimum image solution is obtained. Iterative reconstruction has been widely used in CT because the measurements are typically noisy or a mathematical inverse is unknown or computationally challenging. Examples of iterative reconstruction in CT include the algebraic reconstruction technique (ART) (52) and the simultaneous algebraic reconstruction technique (SART) (46). Iterative reconstruction usually outperforms analytic reconstruction in terms of the quality of reconstructed images, because iterative reconstruction relies on a more improved forward model and has the ability to bring in various types of external prior information to expand the information available during reconstruction.

Very recently, a third type of CT reconstruction method - deep learning based reconstruction - was introduced. Deep learning reconstruction was first introduced to CT in 2016, when Kang et al. used a deep learning reconstruction approach at the 2016 Low-Dose X-ray CT Grand Challenge [organized by the American Association of Physicists in Medicine (AAPM)] (53), and, in parallel, when Chen et al. introduced a similar convolutional neural network (CNN) for low-dose CT denoising (54). The successful demonstration of CNN reconstruction in low-dose CT has inspired many other deep learning reconstruction research. For example, a combination of a CNN with the Normalized Metal Artifact Reduction (NMAR) algorithm for CT metal artifact reduction (55), a combination of DenseNet and Deconvolution Network (DD-Net) for sparseview CT (56), Super-Resolution Convolutional Neural Network (SRCNN) for CT super-resolution (57), and so on.

Deep learning reconstruction does not require an explicit physical imaging model. Instead, deep learning reconstruction can build its own model from a large amount of training data, which becomes more and more readily available due to the wide use of medical imaging in modern healthcare. With larger and more representative training datasets, deep learning reconstruction has the potential to outperform both analytic reconstruction and iterative reconstruction. With unsupervised learning or self-supervised learning, it has been hypothesized that the integration of imaging physics within the machine learning pipeline may further improve the reconstruction quality. For example, a self-supervised and hybrid CT super-resolution model that integrates the advantages of both deep learning network and imaging physics has been just published very recently (51).

\section{PET}

Similarly, PET reconstruction aims to generate diagnostic quality images from measurement data. The conventional PET reconstruction methods can be broadly classified into two categories, i.e., (1) analytic $(58,59)$ and (2) iterative PET reconstruction methods $(60,61)$. The analytic PET reconstruction methods provide a straightforward mathematical solution for image formation, a typical example of which is the filtered-back projection (FBP). In contrast, based on a more accurate description of the imaging process, iterative methods produce a more complex mathematical solution that requires multiple steps to reach an image. Since it can take into account the noise patterns in the observations and use more realistic models of the system, the iterative methods provide improvements over the analytical methods. The classical iterative methods include Maximum Likelihood-Expectation Maximization (ML-EM) (60) and Ordered Subsets Expectation Maximization (OSEM) (61).

Recently, numerous learning-based methods have also been developed for PET reconstruction, such as random forest (62), sparse representation (SR) (63), and multi-level Canonical Correlation Analysis (mCCA) scheme (64). Yet, these traditional machine learning methods often require complex feature engineering, which largely limits the practicability and 
also results in suboptimal reconstruction quality. To address this limitation, deep learning was first introduced to PET reconstruction in 2017 by Xiang et al. (65). The authors proposed a deep CNN model, followed by an auto-context strategy, to estimate standard-dose PET images directly from both the low-dose PET and the corresponding MR images, without the need for handcrafted features. Encouraged by the great success of this work, a series of deep learning-based methods have been developed and successfully applied to various scenarios of PET reconstruction (58-61, 66, 67). In addition, the combination of the conventional iterative reconstruction framework and the deep learning-based method has provided some new approaches for PET reconstruction (14, 68, 69). For instance, Gong et al. (14) used the existing inter-patient information via a deep neural network to further improve the quality of the reconstructed PET image. Furthermore, with the introduction and development of new deep learning models such as GAN, more efforts applying new techniques have been continuously conducted for superior PET reconstruction performance (70-72).

\section{Training and Testing Workflow}

The image reconstruction framework typically includes an input, a reconstruction model, and an output. Traditionally, the input is a sensor-domain raw data, i.e., sinogram in CT. With deep learning-based reconstruction, the sensor data can be first reconstructed using an analytic reconstruction model to provide a low-quality image, and then this low-quality image is fed into the DNN model to generate the corresponding high-quality image. For MRI, the input and output data pair can be either in k-space or image space. Note, to build a deep learning-based reconstruction framework, two steps, namely model training and model testing, are included, as detailed below.

Model training is performed on the provided training samples to optimize the model parameters. During the model training, the loss between the model-generated outputs and the provided training samples is calculated and back-propagated to optimize the model parameters. The model parameters are updated to minimize this loss. Model training proceeds in a data batch mode. Training is stopped after the model is converged to a certain point, or after reaching a pre-selected number of epochs. To avoid the overfitting issue, data augmentation is commonly utilized. Frequently utilized data augmentation methods include affine transformations and Gaussian noise addition. In a deep learning model, there are usually some hyper-parameters (such as batch size, learning rate, etc.) that need to be adjusted manually or automatically, i.e., using an additional validation set, to improve the model performance.

With the optimized model, testing can be performed. To comprehensively evaluate the model performance, testing with data different from the training/validation data should be conducted. For example, validating and testing data from different centers collected with different machines are often considered to make the model robust enough in realworld applications.

\section{TECHNICAL DEVELOPMENTS OF DEEP LEARNING-BASED RECONSTRUCTION}

This section will review various deep learning reconstruction methods developed for MRI, CT, and PET, with typical methods summarized in Table 1. We will present technical aspects and performance characterization of deep learning reconstruction. Technical aspects will include data preparation, network architecture design, loss function, and settings or requirements for training.

\section{Data Preparation}

When applying deep learning to medical imaging, normally three datasets are in need, namely training, validation, and testing datasets. The training dataset is used to train a neural network that is monitored by the validation dataset to avoid overfitting or underfitting. The testing dataset is to evaluate whether the deep learning models can perform well for the real application scenarios. The datasets should include ground-truth images for supervised learning. While for unsupervised learning, no ground-truth information is needed.

For MRI, different types of datasets have been collected and experimented with for various applications. According to the target region dynamic characteristics, there are static MRI and dynamic MRI. Static MRI is applicable when the imaging target changes slowly with time, such as the knee $(36,38)$ and the brain (37). Dynamic MRI is often required when the target moves fast, such as cardiac MRI $(74,91)$. Based on the number of coils utilized to collect the data, MRI datasets can be classified into single-channel MRI (92) and multi-channel MRI (43, 76, 93). When different imaging parameters are used, multi-parametric MRI data are collected to better characterize the physical and physiological properties of the imaging object (94). Besides, quantitative MRI is also available, which can measure tissuespecific parameters $(95)^{1}$.

For CT, depending on the goal of network training, various public datasets are available for DNN model training when developing deep learning reconstruction methods. Some datasets are curated for image noise reduction. For example, the Mayo Clinic Low-Dose X-ray CT datasets for the Low Dose CT Grand Challenge organized by the AAPM (54) have clinical CT images acquired at the full-dose level and the corresponding simulated CT images at the quarter-dose level. This Mayo Clinic dataset can be useful for training deep learning models to reduce CT image noise and hence optimize the dose efficiency. Other datasets are curated toward specific diseases or conditions. For example, The Cancer Imaging Archive (TCIA) hosts a large archive of medical CT images of cancer accessible for public download. Noticeably, in the last year, because CT has been successfully proven to be a rapid triaging tool in patients with moderate to severe COVID symptoms in a resourceconstrained environment where COVID-19 is highly prevalent (96), we now have abundant publicly-available COVID CT datasets available today. Two particular COVID CT datasets

\footnotetext{
${ }^{1}$ Popular datasets in MRI include fastMRI https://fastmri.org/dataset/ and brainweb https://brainweb.bic.mni.mcgill.ca/.
} 
TABLE 1 | Representative works on deep learning-based MRI/CT/PET image reconstruction.

\begin{tabular}{|c|c|c|c|c|c|c|}
\hline Modality & Task description & Network architecture & Loss function & Dataset & $\begin{array}{l}\text { Evaluation } \\
\text { metrics }\end{array}$ & Reference \\
\hline MRI & $\begin{array}{l}\text { Directly learning the } \\
\text { transformation from } \\
\text { sensor-space data to image }\end{array}$ & MLP & $\begin{array}{l}\text { Simple squared loss and } \\
\text { additional L1-norm penalty }\end{array}$ & $\begin{array}{l}\text { ImageNet database, } \\
\text { MGH-USC HCP public } \\
\text { database }\end{array}$ & SNR, RMSE & $(37)$ \\
\hline MRI & $\begin{array}{l}\text { k-space to k-space } \\
\text { reconstruction }\end{array}$ & UNet & L2 loss & $\begin{array}{l}\text { Knee k-space dataset, } \\
\text { MGH-USC HCP public } \\
\text { database }\end{array}$ & $\begin{array}{l}\text { NMSE, PSNR, } \\
\text { SSIM }\end{array}$ & $(36)$ \\
\hline MRI & $\begin{array}{l}\text { Reconstruction with } \\
\text { proposed complex } \\
\text { convolution operations }\end{array}$ & ResNet & Mean absolute error (MAE) & Brain dataset, Knee dataset & PSNR, SSIM & $(43)$ \\
\hline MRI & $\begin{array}{l}\text { Reconstructing real-valued } \\
\text { and complex-valued MRI } \\
\text { data }\end{array}$ & GAN & Cyclic data consistency loss & $\begin{array}{l}\text { IXI database, Data Science } \\
\text { Bowl challenge, Knee } \\
\text { dataset }\end{array}$ & $\begin{array}{l}\text { PSNR, SSIM, } \\
\text { NRMSE }\end{array}$ & $(44)$ \\
\hline MRI & $\begin{array}{l}\text { Fast and high-quality } \\
\text { reconstruction by combining } \\
\text { various loss functions }\end{array}$ & GAN & $\begin{array}{l}\text { Content loss, Image domain } \\
\text { and frequency domain } \\
\text { MSE loss, Perceptual } \\
\text { VGG loss }\end{array}$ & $\begin{array}{l}\text { MICCAI } 2013 \text { grand } \\
\text { challenge dataset, } \\
\text { Pathological MRI images }\end{array}$ & $\begin{array}{l}\text { NMSE, PSNR, } \\
\text { SSIM }\end{array}$ & $(52)$ \\
\hline MRI & $\begin{array}{l}\text { Infusing motion information } \\
\text { into the modeling process } \\
\text { with deep neural networks } \\
\text { for enhanced dynamic MRI } \\
\text { reconstruction quality }\end{array}$ & $\begin{array}{l}\text { Recurrent neural network } \\
\text { (MODRN, Motion-guided } \\
\text { Dynamic Reconstruction } \\
\text { Network) }\end{array}$ & L1 loss & $\begin{array}{l}\text { Private short-axis cardiac } \\
\text { data ( } 21 \text { normal subjects } \\
\text { and } 3 \text { dyssynchrony disease } \\
\text { patients) }\end{array}$ & $\begin{array}{l}\text { NMSE, PSNR, } \\
\text { SSIM }\end{array}$ & (73) \\
\hline MRI & $\begin{array}{l}\text { Reconstruction with both } \\
\text { k-space and spatial prior } \\
\text { knowledge integrated via } \\
\text { multi-supervised network } \\
\text { training }\end{array}$ & CNN & L2 loss & Private cardiac MR data & $\begin{array}{l}\text { MSE, PSNR, } \\
\text { SSIM }\end{array}$ & $(74)$ \\
\hline MRI & $\begin{array}{l}\text { Improving MRI } \\
\text { reconstruction accuracy } \\
\text { and computational speed } \\
\text { with a CS-based model }\end{array}$ & $\begin{array}{l}\text { Model-based (Alternating } \\
\text { direction method of } \\
\text { multipliers algorithm) }\end{array}$ & NMSE & Brain and chest MR images & $\begin{array}{l}\text { NMSE, PSNR, } \\
\text { Test time }\end{array}$ & $(40)$ \\
\hline MRI & $\begin{array}{l}\text { Fast and high-quality } \\
\text { reconstruction of clinical } \\
\text { accelerated multi-coil MR } \\
\text { data }\end{array}$ & $\begin{array}{l}\text { Model-based (Variational } \\
\text { network, unrolling iteration) }\end{array}$ & MSE & Clinical knee dataset & SSIM, NRMSE & (38) \\
\hline MRI & $\begin{array}{l}\text { Deriving deep architectures } \\
\text { for inverse problems with } \\
\text { the arbitrary structure }\end{array}$ & $\begin{array}{l}\text { Model-based (recursive } \\
\text { framework alternating } \\
\text { between denoising block } \\
\text { and data-consistency layer) }\end{array}$ & MSE & $\begin{array}{l}\text { Brain MR dataset from five } \\
\text { volunteers }\end{array}$ & PSNR, Time & $(75)$ \\
\hline MRI & $\begin{array}{l}\text { Fast parallel MR imaging by } \\
\text { exploring both spatial } \\
\text { redundancy and multi-coil } \\
\text { correlations }\end{array}$ & $\begin{array}{l}\text { Model-based (split Bregman } \\
\text { iterative algorithm) }\end{array}$ & MSE & $\begin{array}{l}\text { Private } 2 \mathrm{D} \text { multichannel MR } \\
\text { brain dataset }\end{array}$ & $\begin{array}{l}\text { NMSE, PSNR, } \\
\text { SSIM }\end{array}$ & $(76)$ \\
\hline MRI & $\begin{array}{l}\text { Self-supervised deep } \\
\text { learning MRI reconstruction } \\
\text { by dividing sub-sampled } \\
\text { data points into two sets } \\
\text { with one for data } \\
\text { consistency and another for } \\
\text { loss calculation }\end{array}$ & $\begin{array}{l}\text { Model-based (regularized } \\
\text { iterative algorithm between } \\
\text { data consistency and a } \\
\text { regularizer solved by the } \\
\text { variable-splitting and } \\
\text { quadratic relaxation method) }\end{array}$ & Normalized L1-L2 loss & $\begin{array}{l}\text { Knee MR data from fastMRI } \\
\text { initiative database }\end{array}$ & NMSE, SSIM & $(77)$ \\
\hline MRI & $\begin{array}{l}\text { Accelerate and improve } \\
\text { multishot diffusion-weighted } \\
\text { MRI reconstruction by } \\
\text { combining unrolled network } \\
\text { with deep CNNs }\end{array}$ & $\begin{array}{l}\text { Model-based and UNet } \\
\text { (recurrences of } \\
\text { model-based gradient } \\
\text { updates (shotlocally } \\
\text { low-rank) and neural } \\
\text { networks }\end{array}$ & L1 loss & $\begin{array}{l}\text { Private brain ( } 14 \text { scans from } \\
8 \text { volunteers) and breast ( } 6 \\
\text { scans from } 6 \text { volunteers) } \\
\text { MR data }\end{array}$ & $\begin{array}{l}\text { NMSE, PSNR, } \\
\text { SSIM }\end{array}$ & (78) \\
\hline CT & $\begin{array}{l}\text { Using U-Net and its variants } \\
\text { for recovery of } \\
\text { high-frequency edges in } \\
\text { sparse-view CT in the image } \\
\text { domain }\end{array}$ & $\begin{array}{l}\text { Dual frame and tight frame } \\
\text { U-Nets }\end{array}$ & $\begin{array}{l}\text { Pixel-wise soft-max } \\
\text { combined with cross } \\
\text { entropy function (the original } \\
\text { U-net loss function) }\end{array}$ & $\begin{array}{l}10 \text { patient CT scan data } \\
\text { from the } 2016 \text { AAPM Low } \\
\text { Dose CT Grand Challenge } \\
\text { Dataset }\end{array}$ & $\begin{array}{l}\text { NMSE, PSNR, } \\
\text { SSIM }\end{array}$ & $(56)$ \\
\hline
\end{tabular}


TABLE 1 | Continued

\begin{tabular}{|c|c|c|c|c|c|c|}
\hline Modality & Task description & Network architecture & Loss function & Dataset & $\begin{array}{l}\text { Evaluation } \\
\text { metrics }\end{array}$ & Reference \\
\hline CT & $\begin{array}{l}\text { General sparse-view CT } \\
\text { image reconstruction }\end{array}$ & $\begin{array}{l}\text { DenseNet combined with } \\
\text { deconvolution }\end{array}$ & $\begin{array}{l}\text { Weighted loss between } \\
\text { MSE and MS-SSIM }\end{array}$ & $\begin{array}{l}\text { 3,059 clinical CT images } \\
\text { from the TCIA database }\end{array}$ & $\begin{array}{l}\text { MSE, SSIM, } \\
\text { Haralick texture } \\
\text { features }\end{array}$ & (15) \\
\hline CT & CT super-resolution & Modified U-net & L2 loss & 7,670 CT slices & NRMSE, PSNR & (57) \\
\hline CT & $\begin{array}{l}\text { Low-dose CT for mapping } \\
\text { low-dose images to } \\
\text { normal-dose images; CT } \\
\text { image denoising }\end{array}$ & $\begin{array}{l}\text { Residual encoder-decoder } \\
\text { CNN (RED-CNN) }\end{array}$ & MSE loss & $\begin{array}{l}\text { 7,015 normal-dose CT } \\
\text { images from the NBIA } \\
\text { dataset and simulated } \\
\text { low-dose CT images }\end{array}$ & $\begin{array}{l}\text { RMSE, PSNR, } \\
\text { SSIM }\end{array}$ & (54) \\
\hline CT & CT image denoising & $\begin{array}{l}\text { Framelet-based wavelet } \\
\text { residual network }\end{array}$ & $\begin{array}{l}\text { Pixel-wise soft-max } \\
\text { combined with cross } \\
\text { entropy function (the original } \\
\text { U-net loss function) }\end{array}$ & $\begin{array}{l}10 \text { patient CT scan data } \\
\text { from the } 2016 \text { AAPM Low } \\
\text { Dose CT Grand Challenge } \\
\text { Dataset }\end{array}$ & $\begin{array}{l}\text { RMSE, PSNR, } \\
\text { SSIM }\end{array}$ & (79) \\
\hline CT & $\begin{array}{l}\text { Sparse-view CT image } \\
\text { reconstruction }\end{array}$ & $\begin{array}{l}\text { U-net with skip connection } \\
\text { for residual learning }\end{array}$ & $\begin{array}{l}\text { Pixel-wise soft-max } \\
\text { combined with cross } \\
\text { entropy function (the original } \\
\text { U-net loss function) }\end{array}$ & $\begin{array}{l}\text { The } 2016 \text { AAPM Low Dose } \\
\text { CT Grand Challenge } \\
\text { Dataset, plus } 500 \text { simulated } \\
\text { images and } 377 \\
\text { experimental sinograms }\end{array}$ & SNR & (80) \\
\hline CT & $\begin{array}{l}\text { CT image denoising in } \\
\text { low-dose CT }\end{array}$ & $\begin{array}{l}\text { GAN network, consisting of } \\
\text { a Generator } \mathrm{CNN} \text { and a } \\
\text { Discriminator } \mathrm{CNN}\end{array}$ & $\begin{array}{l}\text { binary cross-entropy, L2 } \\
\text { loss }\end{array}$ & $\begin{array}{l}5 \text { low-dose and } 5 \\
\text { corresponding routine-dose } \\
\text { CT scans of a phantom, } \\
\text { and } 28 \text { cardiac CT scans } \\
\text { from patients }\end{array}$ & SNR, PSNR & (81) \\
\hline CT & $\begin{array}{l}\text { CT image denoising in } \\
\text { low-dose CT }\end{array}$ & $\begin{array}{l}\text { GAN network with } \\
\text { Wasserstein distance and } \\
\text { perceptual loss (WGAN) }\end{array}$ & $\begin{array}{l}\text { Wasserstein distance based } \\
\text { adversarial loss, VGG } \\
\text { perceptual loss }\end{array}$ & $\begin{array}{l}10 \text { patient CT scan data } \\
\text { from the } 2016 \text { AAPM Low } \\
\text { Dose CT Grand Challenge } \\
\text { Dataset }\end{array}$ & PSNR, SSIM & (82) \\
\hline CT & CT super-resolution & GAN-CIRCLE & $\begin{array}{l}\text { Adversarial loss, cycle } \\
\text { consistency loss, identity } \\
\text { loss, joint sparsifying } \\
\text { transform loss }\end{array}$ & $\begin{array}{l}\text { Micro-CT dataset from } 25 \\
\text { tibia specimen, and the } \\
2016 \text { AAPM Low Dose CT } \\
\text { Grand Challenge Dataset }\end{array}$ & $\begin{array}{l}\text { PSNR, SSIM, } \\
\text { IFC }\end{array}$ & (83) \\
\hline CT & CT Super-resolution & $\begin{array}{l}\text { Self-supervised SADIR-net } \\
\text { (super-resolution and deblur } \\
\text { based iterative } \\
\text { reconstruction), which is a } \\
\text { hybrid between deep } \\
\text { learning network and } \\
\text { imaging physics }\end{array}$ & $\begin{array}{l}\text { Joint loss function } \\
\text { combining L2-norm with } \\
\text { SSIM }\end{array}$ & $\begin{array}{l}47 \text { clinical CT scans from } \\
\text { TCIA database; } \\
\text { custom-acquired } \\
\text { Catphan }{ }^{700} \text { phantom CT } \\
\text { sensor data }\end{array}$ & $\begin{array}{l}\text { MTF, RMSE, } \\
\text { SSIM, IFC }\end{array}$ & (51) \\
\hline PET & $\begin{array}{l}\text { Incorporating the neural } \\
\text { network into the iterative } \\
\text { PET reconstruction } \\
\text { framework for PET } \\
\text { denoising }\end{array}$ & UNet with residual learning & $\begin{array}{l}\text { Augmented Lagrangian } \\
\text { format, L2 loss }\end{array}$ & $\begin{array}{l}19 \text { XCAT phantoms; } \\
6 \text { lung patient data }\end{array}$ & CR, STD & (14) \\
\hline PET & $\begin{array}{l}\text { Standard-dose PET } \\
\text { reconstruction from } \\
\text { low-dose PET }\end{array}$ & Noise-Aware Dual Res-UNet & $\begin{array}{l}\text { Dice loss, Binary cross } \\
\text { entropy loss, General and } \\
\text { adaptive robust loss, SSIM } \\
\text { loss }\end{array}$ & $\begin{array}{l}10 \text { subjects referred for } \\
\text { whole-body FDG-18 } \\
\text { PET/CT scan on a GE } \\
\text { Discovery } 710 \text { scanner }\end{array}$ & PSNR, SSIM & (85) \\
\hline PET & $\begin{array}{l}\text { Using patients' own prior } \\
\text { information for PET } \\
\text { reconstruction }\end{array}$ & 3D UNet & MSE & Phantom and real brain data & CRC, STD & (69) \\
\hline PET & $\begin{array}{l}\text { PET reconstruction from } \\
\text { projections data }\end{array}$ & ANN & MSE & Simulated data & NMSE & (86) \\
\hline
\end{tabular}


TABLE 1 | Continued

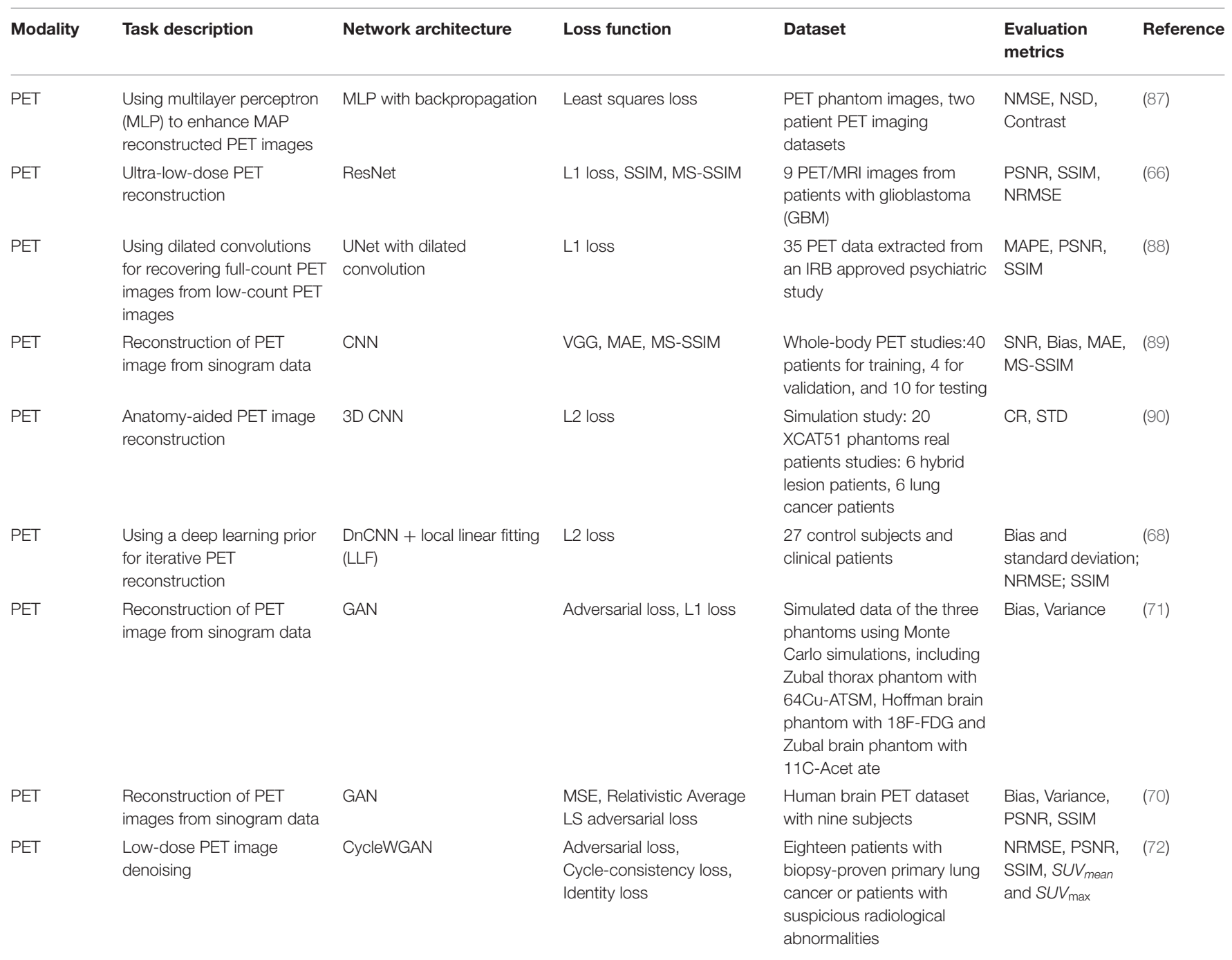

could be useful for training deep learning models. One is the BIMCV-COVID-19+ (97), a large dataset from the Valencian Region Medical Image Bank earlier in the pandemic period, and another is the RSNA International COVID-19 Open Radiology Database (RICORD), which is an ongoing international effort in curating potentially the largest international COVID-19 CT dataset.

For PET, the datasets mainly include static PET (98-100) and dynamic PET (101-104) based on data types. On the other hand, according to the number of tracers imaged in a single scan, the datasets can be classified as single-tracer PET (105), dual-tracer PET $(106,107)$, and multi-tracer PET (108). When it comes to the injected tracer dose level, the datasets can also be broadly categorized as low-dose PET (L-PET) and full-dose PET (F-PET) $(65,67,88)$. Although the use of real PET data in studies is more clinically relevant, these real data are often difficult to obtain due to various factors. Therefore, simulated phantom data is becoming a popular alternative in research works $(68,88,109,110)$.

\section{Network Architecture}

The neural network architectures employed for different tomographic imaging tasks share some similar properties. The most frequently used architectures include multilayer perceptron (MLP), U-Net, generative adversarial networks (GAN), ResNet, etc. Here, we introduce these typical network architectures.

\section{MLP}

The MLP, which is an artificial neural network (ANN) with all layers fully-connected, can map sets of input data into a set of desired outputs. In the past decades, researchers have worked on exploiting MLP in medical image analysis. For example, a multilayer perceptron was proposed for accelerated parallel MRI (111). Zhu et al. (37) proposed an MLP-based manifold learning framework to emulate the fast-Fourier transform and learn an end-to-end mapping between k-space data and image domains and achieve the purpose of acceleration. For PET, MLP was also employed for simple low-resolution PET reconstruction (86). Furthermore, Yang et al. (87) developed an MLP-based 
framework to enhance the maximum a posteriori (MAP) reconstructed PET images, which constructs a highly non-linear and spatial-varying mapping between the MAP reconstructed image patches and the corresponding enhanced image patches.

\section{U-Net}

U-net consists of an encoder structure and a decoder structure, which was originally designed for biomedical image segmentation $(112,113)$. The encoder gradually down samples the input images to extract image features with different levels of semantic information. The decoder receives the features from the encoder and recovers the feature map resolution step-by-step to generate the outputs, which are often the same size as the inputs and can then be treated as the reconstructed images. Skip connections between the encoder and the decoder are introduced to improve the localization accuracy during decoding.

For MRI, Ye et al. (114) used deep residual learning to accelerate MRI. The proposed deep residual learning network is composed of two separately trained amplitude and phase difference networks, which can successfully learn and remove aliasing artifacts. Furthermore, Ye et al. also proposed a UNet-based domain adaptation architecture for radial k-space undersampled MR (115), and a fully data-driven deep learning algorithm for $\mathrm{k}$-space interpolation (36). These methods have been successfully applied to MR image reconstruction, and have achieved better results than the classic CS method. Duan et al. (116) proposed a fast and accurate deep learning reconstruction method for human lung gas MRI, which consists of coarse-tofine nets (C-net and F-net) based on U-Net. The proposed deep learning method can better reconstruct the human lung gas MR images acquired from highly undersampled k-space compared with the traditional CS-MRI. Hyun et al. (117) proposed an under sampling MRI reconstruction method using U-Net, which shows excellent performance and can generate high-quality MR images with a small amount of data.

For CT, U-net and its variants have also been successfully applied to solve various problems in CT reconstruction, including sparse-view CT reconstruction, artifact reduction, noise suppression, and CT super-resolution, etc. For sparseview CT reconstruction, which can reduce radiation dose and accelerate scanning speed, Han et al. (56) achieved better reconstruction performance by framing U-Net via deep convolutional framelets. Also, for sparse-view CT reconstruction, Kofler et al. (118) proposed a cascade of U-nets and data consistency layers, and Zhang et al. (15) developed DD-Net by combining DenseNet and deconvolution and arranging them in a network topology similar to U-Net. For the purpose of CT artifact reduction, Zhang et al. (55) tried U-net and found promising results of $\mathrm{U}$-net in reducing global and local CT artifacts. To reduce noise in low-dose CT images, Liu et al. (119) adopted stacked denoising autoencoders to suppress noise and recover structure details. For CT super-resolution, Park et al. (57) used a modified U-net to learn an end-to-end mapping between lowresolution and high-resolution CT images.

For PET, U-net is also a commonly used framework in many PET reconstruction works $(14,69,86,120-122)$. Gong et al. (14) designed an iterative reconstruction framework that combines the U-net structure and the residual network for PET denoising by utilizing dynamic data of prior patients. Taking the noise level of low-count PET into account, Xiang et al. (85) developed a noise-aware dual Res-UNet (NADRU) framework for low-dose PET reconstruction. The proposed method first identified an attention map indicating the location of high-intensity noise in the low-dose PET images. Then, the noise attention map was incorporated with the original image for high-quality PET reconstruction. In addition to reconstructing high-quality images within PET, many efforts have also been made to reconstruct PET from other modalities. For example, Sikka et al. (121) adopted a 3D U-Net architecture to estimate PET from MRI images. By considering non-local and non-linear correlations, the proposed method showed a significant improvement in the diagnostic accuracy of Alzheimer's disease. Employing a modified 3D U-net as the network structure, Gong et al. (69) designed an iterative reconstruction framework that incorporates the personalized deep neural network to generate PET data from a patient's own MRI prior image(s). Furthermore, Cui et al. (122) utilized CT/MR prior information to perform PET denoising based on a modified 3D U-net structure in an unsupervised manner.

\section{ResNet}

ResNet is proposed to solve the difficulty of training very deep CNNs and avoid model performance degradation (123). The core idea of ResNet lies in residual learning, which is based on the assumption that it is easier to optimize the residual mapping than to optimize the original and unreferenced mapping (123). With the success of residual learning, the ResNet has also been widely used in medical image reconstruction.

For MRI, Shi et al. $(124,125)$ proposed a residual-learningbased MR image super-resolution reconstruction network. The network can improve image reconstruction performance using both global residual learning (GRL) and local residual learning (LRL). Wang et al. (43) proposed a new framework Deepcomplex MRI using a deep residual CNN for parallel imaging. It considers the correlation between the real and imaginary parts of $\mathrm{MR}$ complex images and achieved better results than real-value networks. Li et al. (126) designed a deep ResNet using variable density spiral trajectory to accelerate fMRI reconstruction. The proposed deep ResNet consists of various residual blocks. $\mathrm{Du}$ et al. (127) proposed a residual CNN for reconstructing single anisotropic 3D MR images based on residual learning. The residual $\mathrm{CNN}$ with long and short skip connections can effectively recover uncollected high-frequency details of MR images.

For CT, ResNet or more generally residual learning has also been demonstrated its effectiveness in CT reconstruction, particularly in noise suppression and artifact reduction. Chen et al. (128) developed a residual encoder-decoder CNN (RED$\mathrm{CNN}$ ) for low-dose CT. RED-CNN combines autoencoder, deconvolution network, and shortcut connections. It can effectively suppress noise, preserve structure details, and enhance lesion detection. For CT image denoising, Kang et al. (79) proposed a wavelet residual network based on a deep convolutional framelet and achieved better performance compared to their earlier algorithm using directional deep 
convolutional-wavelet neural network (53). To reduce the sparseview CT artifact, Dong et al. (129) proposed a residual deep learning $\mathrm{CNN}$ to interpolate the sinogram of sparse-view micro$\mathrm{CT}$, and the deep learning interpolated sinogram was FBPreconstructed into high-quality images. Also for sparse-view CT, Jin et al. (81) proposed FBPConvNet, which first reconstructs sparse-view CT sinogram with FBP and then improves the FBPreconstructed image using a modified $U$-net with the addition of residual learning.

For PET, residual learning is also employed in the reconstruction task. In order to effectively restore the lowdose PET images to the standard-dose quality, $\mathrm{Xu}$ et al. (66) proposed an encoder-decoder residual deep network, in which residual learning and skip connections were adopted for learning the difference between standard-dose and low-dose PET images. Similarly, Spuhler et al. (88) designed a novel multiscale dilated CNN approach to predict full-count PET images from low-count images. The proposed method integrated the residual learning to capture the difference of low-count and full-count PET images and enhance the convergence of the network. The experiments of these studies showed that residual learning was beneficial for high-quality PET reconstruction. Moreover, in Chen et al. (54), a deep learning-based framework with low-count PET and multimodal MRI as inputs was presented for diagnostic-quality PET image synthesis through residual learning.

\section{GAN}

GAN (130), as one of the most popular generative models in deep learning, has demonstrated its superior performance in many computer vision tasks and attracted growing interest in medical image reconstruction.

For MRI, Yang et al. (52) proposed De-Aliasing GAN (DAGAN) for fast compressed sensing MRI reconstruction. The authors designed a refinement learning method to stabilize the UNet-based generator. In order to better preserve texture and edge information, DAGAN combines adversarial loss and innovative content loss in the image reconstruction process and takes into account the frequency information at the same time. The reconstruction result of DAGAN is better than the traditional CSMRI algorithm. Quan et al. (44) proposed an improved model, RefineGAN, based on fully residual convolutional autoencoder and GANs for fast and accurate CS-MRI reconstruction. It can perform faithful interpolation for a given undersampled kspace data by employing a deeper generator and discriminator with cyclic data consistency loss. RefineGAN outperforms the state-of-the-art CS-MRI reconstruction algorithms in terms of both image quality and running time. Mardani et al. (131) proposed a novel CS framework based on LSGAN and pixelwised 11/12 loss for MRI reconstruction, namely GANCS. GANCS can reconstruct higher quality images with improved fine texture details compared to existing methods.

For CT, Wolterink et al. (81) used a GAN network that consists of a Generator $\mathrm{CNN}$ and a Discriminator $\mathrm{CNN}$ to reduce the noise level in CT images. They produced better images for more accurate coronary calcium quantification. Similarly, for the purpose of image denoising in low-dose CT, Yang et al. (82) modified the original GAN network by using the Wasserstein distance, instead of the Jensen-Shannon (JS) divergence, to compare data distributions. The Wasserstein distance is combined with the well-known pre-trained VGG-19 network (132) to build a joint loss function. This modified GAN network also achieved promising results in image denoising. For the purpose of CT super-resolution, You et al. (83) developed a GAN network constrained by the identical, residual, and cycle learning ensemble (GAN-CIRCLE). GAN-CIRCLE incorporates deep $\mathrm{CNN}$, residual learning, and network-innetwork techniques for feature extraction and restoration, and employed a cycle Wasserstein regression adversarial training framework. It is noted that many GAN networks also employed the technique of residual learning in their architectures.

For PET, Liu et al. (71) employed a conditional GAN (cGAN) framework to learn the mapping from sinogram data to reconstructed PET images directly. Inspired by the promising results achieved by cGAN, the authors further presented an end-to-end model for PET reconstruction, which adopts two coupled networks to sequentially denoise low dose sinogram and reconstruct activity map (70). Zhou et al. (72) designed a cycle Wasserstein regression adversarial model (CycleWGAN) using Wasserstein distance, instead of JS divergence and cycle-loss, to boost the low-dose PET image quality, which shows the superior performance of Wasserstein distance in effectively preserving the edge information. To reduce the loss of contextual information, Wang et al. (133) developed a concatenated 3D cGAN for highquality PET image estimation from low count PET. Considering the various contributions of different image locations and the complementary information in different modalities, they further proposed an auto-context-based locality adaptive GANs (LAGANs) (67) model to reconstruct the full count PET image from both the low count PET and the accompanying multimodal MRI images. Besides, many other works also attempted to reconstruct PET images from other modality information in consideration of the expensive cost of PET imaging and the hazards of radiation exposure. Ben-Cohen et al. (134) proposed to generate simulated PET images from given CT data without manually annotated labels. They first adopted FCN to generate an initial PET-like image and then employed cGAN to refine the FCN output so that the synthesized image could be more realistic. Based on 3D GAN, Yaakub et al. (135) designed a twostage approach to predict accurate PET images from T1-weighted MRI scans. It is worth noting that many GAN-based models have also introduced residual learning to further improve the reconstruction performance $(136,137)$.

\section{Modality-Specific Module Design}

To improve the reconstruction accuracy or enhance the reliability of the reconstruction results, special network modules are usually designed taking the specific properties of different imaging modalities into consideration.

For MRI, in addition to modules utilized by every model, including the convolutional layers, the normalization layers, and the activation layers, there is commonly a data consistency layer to guarantee that the data on scanned points are correct (138). According to the data acquisition process of MRI, undersampling happens in the $\mathrm{k}$-space by neglecting a certain portion of data 
points. Therefore, theoretically, on the scanned data points, the reconstruction results should be consistent with the acquisitions. With the data consistency layer, the reconstruction is forced to be correct on these sampling points and the reconstruction of unscanned data points is accordingly improved. Besides, because the data acquisition of MRI proceeds in a different domain ( $\mathrm{k}$ space) from the image domain, reconstruction can be performed in individual domains $(38,139)$ or cross-domains $(74,140)$. Furthermore, complex-valued neural networks are proposed to specifically process the complex-valued MR data $(43,141,142)$.

For CT, although the reconstruction results from most reported deep learning algorithms are so far remarkable in terms of image quality, there is still some concern about whether those reconstruction results can be trusted, especially in real-world applications of diagnostic imaging. One main limitation of those deep learning algorithms is that they seldom provide guarantees in the worst-case scenario. To address this limitation, Gupta et al. (84) proposed a specially designed CT image reconstruction method that replaces the projector in a projected gradient descent with a CNN and uses the CNN in the feedback loop to recursively project the result onto the sensor domain. This reconstruction method can enforce measurement consistency, is guaranteed to converge, and, under certain conditions, converges to a local minimum of a nonconvex inverse problem. On the other hand, while iterative CT reconstruction can yield high-quality images, careful tuning of hyper-parameters in these iterative reconstruction problems is inevitable. To achieve automatic parameter tuning, Shen et al. (143) employed deep reinforcement learning to train a system that can automatically adjust parameters in a humanlike manner, and demonstrated that CT images reconstructed from their approach attain quality similar or better than those reconstructed with manually tuned parameters.

For PET, some studies have incorporated specially designed modules to improve the PET image quality. For instance, taking the location-varying contributions from different imaging modalities into account, Wang et al. (67) proposed a locality adaptive fusion module to automatically fuse local patches from multimodal MRI for high-quality PET image synthesis. In Samuel Matej et al. (58), the authors devised a novel Radon inversion layer to address the computational challenges in multi-slice PET image reconstruction. This specially designed layer was demonstrated to be efficient in performing domain transformation from sinogram to image space. Moreover, to encourage feature reuse and prevent resolution degradation, $\mathrm{Du}$ et al. (144) designed residual dense connections followed with pixel shuffle operations (RDPS blocks) in the generator network, achieving promising reconstruction results.

\section{Loss Function}

As the task is to restore the quality of the output images in all locations, for the fully supervised learning, the most frequently used loss for the network training is the mean squared error (MSE) between the network prediction and the ground truth. MSE is also known as the L2 loss. Based on MSE, there are also some extended loss functions such as root mean squared errors (RMSE), normalized mean squared errors (NMSE), and normalized root mean squared errors (NRMSE).

There are alternative losses, such as the mean-absolute-error cost function (MAE), which is also known as the L1 loss. Compared with MSE, MAE is used relatively less, but there are still studies showing that using MAE can preserve better results than MSE.

One common choice of loss function for reconstruction problem is L2, but the reconstructed image obtained is of low quality and lacks high-frequency detail. Therefore, in order to offset the shortcoming of L2 loss, structural similarity index (SSIM), signal to noise ratio (SNR), peak SNR (PSNR), or perceptual loss is used as an additional loss to constrain the prediction results in some literatures. These additional loss functions or the combined loss between them have been shown to improve the reconstruction performance of the model.

\section{Modality-Specific Loss}

In MRI, there are also specially designed losses. In Quan et al. (44), the authors proposed a cyclic data consistency loss, which combines the undersampled frequency loss and the fully reconstructed image loss. In practice, MSE, MAE or other functions can be used as the basic function to achieve cyclic loss. Some studies (52) combine MSE and perceptual loss to form a novel content loss to achieve better reconstruction details. There are also studies that combine MAE with perceptual loss (145), or MSE with TV loss (146), for MR image reconstruction.

In CT, Yang et al. (84) employed for their modified GAN network a joint loss function that combines the Wasserstein distance-based adversarial loss with the well-known pre-trained VGG-19 loss (134). Those two loss terms in the joint loss function are balanced with a hyperparameter to control the tradeoff between the GAN adversarial loss and the VGG perceptual loss. When comparing the performance of a modularized deep neural network to commercial algorithms for low-dose CT image reconstruction, Shan et al. (147) chose a composite loss function that includes three components: adversarial loss, MSE, and edge incoherence. The adversarial loss is used to train the generator in their GAN network to produce images as close to the reference high-dose images as possible, the MSE is used to reduce image noise, and the edge incoherence is used to enhance the edge information in the denoised image.

In PET, Kim et al. (68) proposed a novel 3D local linear fitting (LLF) function and incorporated it into the cost function, combining the input image with the DnCNN correcting the unwanted bias and finally enhance the image quality. Similarly, Ouyang et al. (105) designed a GAN model with feature matching technique and task-specific perceptual loss to ensure that the synthesized standard-dose amyloid PET images include the correct features.

\section{Requirement for Network Training}

The fundamental parameter learning schemes are backpropagation algorithms. Adam optimization with variable parameter momentum is often used in neural network optimization. As for hardware, the graphics card for deep learning network training is essential. According to the literature 
we searched and referenced, the types of graphics cards generally used are NVIDIA K80, NVIDIA K40c, GTX 1080Ti, RTX 2080, RTX 2080Ti, Titan X, Titan Xp, Titan V, etc. As for software, TensorFlow, PyTorch, Keras, Caffe, etc. are several commonlyused DNN training frameworks. In addition, Matlab is also used to process data or perform tests in some studies. The system used is generally a Linux system.

\section{CLINICAL APPLICATIONS AND CURRENT ACHIEVEMENTS}

Deep learning-based medical imaging techniques have played more and more important roles in today's clinical applications, and have achieved significant progress in solving various major pain points in different imaging modalities.

\section{MRI}

For MRI, it has superior soft-tissue contrast and it is radiationfree. However, the major limitation of MRI is its slow acquisition speed. Although lots of acceleration strategies were proposed in the literature, such as parallel imaging and compressed sensing, they have their own limitations such as amplification of Gibbs artifacts and long iterative reconstruction time. Deep learning-based techniques offer a feasible solution to robustly and efficiently reconstruct the MRI images from subsampled Kspace data even under high down sampling factors. Moreover, deep learning-based reconstruction techniques can be integrated with conventional acceleration techniques to reach even higher reconstruction quality. For instance, the AI-assisted compressed sensing (ACS) technique developed by United Imaging Intelligence (UII) and United Imaging Healthcare (UIH) integrates the advantages of four acceleration techniques, i.e., (1) deep learning-based reconstruction, (2) partial Fourier transform, (3) parallel imaging, and (4) compressed sensing, into a unified framework, and achieves great success in real-world clinical applications for fast MRI imaging. ACS is able to reduce around $80 \%$ scan time on average for most of the FSE sequences, and it supports the scan of different body parts such as head, cervical spine, lumbar spine, hip, pelvis, ankle, and knee. For each body part, ACS normally can achieve a scan time of fewer than $100 \mathrm{~s}$ for all the sequences as shown in Figure 1.

ACS has received FDA 510K clearance and has also been deployed in different hospitals. Another example is the SubtleMR techniques developed by Subtle Medical, which also adopts deep learning-based techniques for fast MR imaging and received FDA $510 \mathrm{~K}$ clearance. SubtleMR is able to reduce around $60 \%$ scan time and has also been deployed in many hospitals and applied in real-world clinical workflow in the US.

\section{CT}

For modalities of CT (as well as PET as introduced below), the radiation dose delivered to the patient must be strictly controlled, because radiation is harmful to the patient and an excessive dose may lead to the result of secondary cancer. However, a lower dose normally leads to inferior image quality, and it may affect the diagnosis accuracy. Therefore, how to obtain high-quality images under the low-dose condition for CT is essential in real world clinical applications.

Deep learning-based denoising techniques provide a good solution to obtain high-quality CT images under low-dose conditions. The basic principle is to train a deep learning network that learns the mapping between the low dose CT image and the corresponding standard-dose CT image. Once the network is trained, the image quality can be significantly improved by passing the low dose CT image through the network. This strategy has been adopted by many industries and turned into products in real world applications. For instance, the DELTA (i.e., DEep Learning Trained Algorithms), a deep learning-based denoising technique developed by UII and UIH, can reduce the dose up to $80 \%$ while the low contrast detectability (LCD) of CT images can be improved up to $157 \%$; some typical examples are shown in Figure 2. Canon developed the Advanced intelligent Clear-IQ Engine (AiCE) which can reduce the noise and boost signal in CT images based on deep learning. GE developed the TrueFidelity CT imaging platform, which adopts deep learningbased techniques to improve the image quality of low-dose CT images. DELTA, AiCE, and TrueFidelity all received FDA $510 \mathrm{~K}$ clearance.

\section{PET}

For PET, besides the concern of dose, another pain point is the relatively longer imaging time than other image modalities such as CT and DR, and some patients such as children and patients with bone cancer may not be able to hold their positions during the imaging process. Therefore, how to obtain high-quality images under low-dose conditions and how to accelerate the imaging is essential for real-world clinical applications of PET.

So far, various deep learning-based techniques have been applied to accelerate the acquisition speed of PET imaging and also maintain the high quality of PET images. For instance, the HYPER DLR (Deep-Learning Reconstruction) product developed by UII and UIH can significantly reduce the scanning time of PET imaging from $3 \mathrm{~min} / \mathrm{bed}$ to $<1 \mathrm{~min} / \mathrm{bed}$. In addition, it can effectively reduce the noise level of PET images under low count rate conditions and significantly improve image quality. Specifically, the SNR (Signal-to-Noise Ratios) of PET images can be improved by $42 \%$ with an accelerated imaging speed. Figure 3 shows some typical examples of HYPER DLR. Similarly, Subtle Medical developed the SubtlePET product which also adopts deep learning-based techniques and can denoise the lowcount PET images obtained in $25 \%$ of the original scan duration, improving patient comfort during PET scans. Both HYPER DLR and SubtlePET received FDA 510K clearance.

\section{PET-MRI}

In some applications, cross-modality synthesis techniques are also required. For instance, the PET-MR imaging equipment normally needs to synthesize the CT image from the acquired MR image in order to perform attenuation correction (AC) for the PET image (148). This process is illustrated in Figure 4.

There are lots of synthesis strategies. The most simple and straightforward strategy is to segment the MR image into several tissue types and fill the corresponding regions with fixed CT HU 

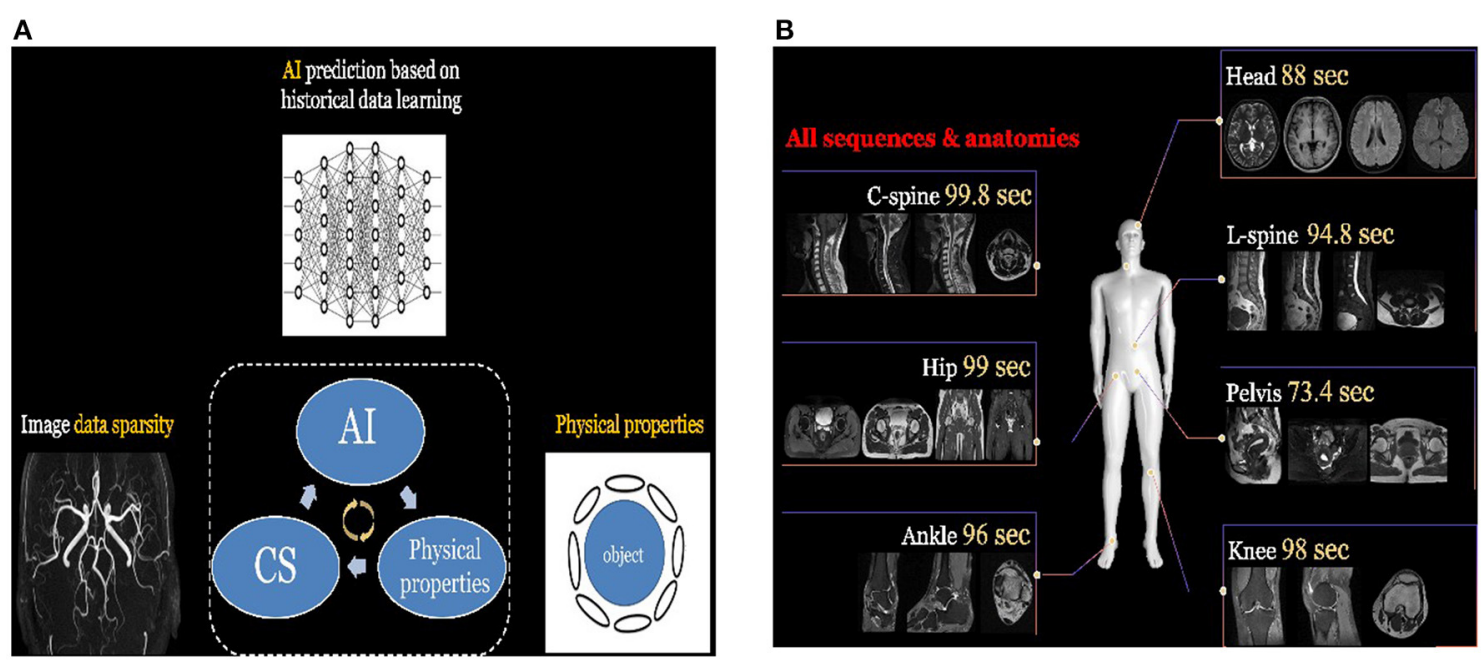

FIGURE 1 | (A) The principle of ACS. It integrates the advantages of different acceleration techniques such as deep learning-based reconstruction, parallel imaging, and compressed sensing. (B) ACS normally can achieve great scan speed (i.e., <100 s) for different body parts and sequences.
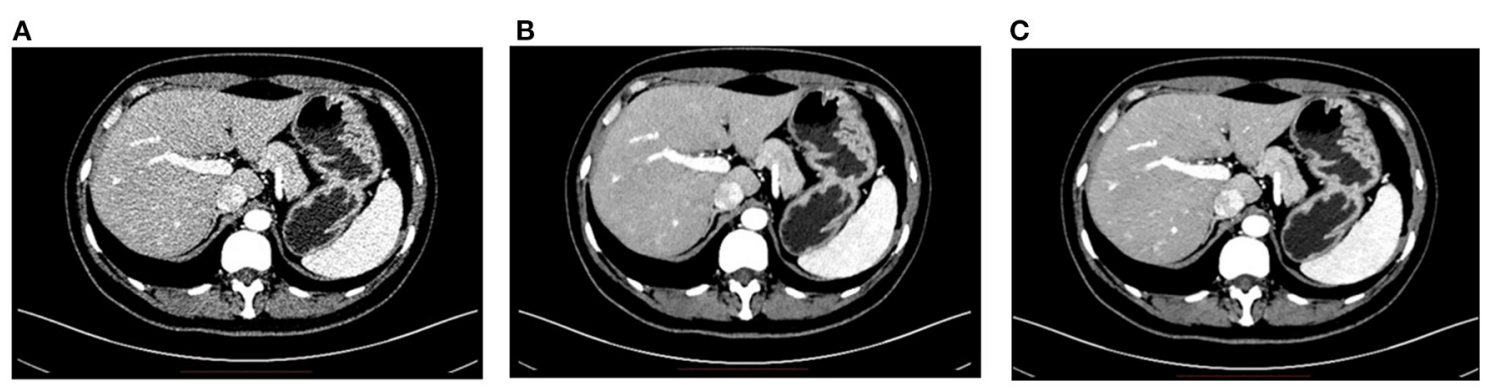

FIGURE 2 | (A) The low dose abdominal CT image. (B) The resulting image after applying DELTA to the low dose image in (A). (C) The corresponding standard dose abdominal CT image.

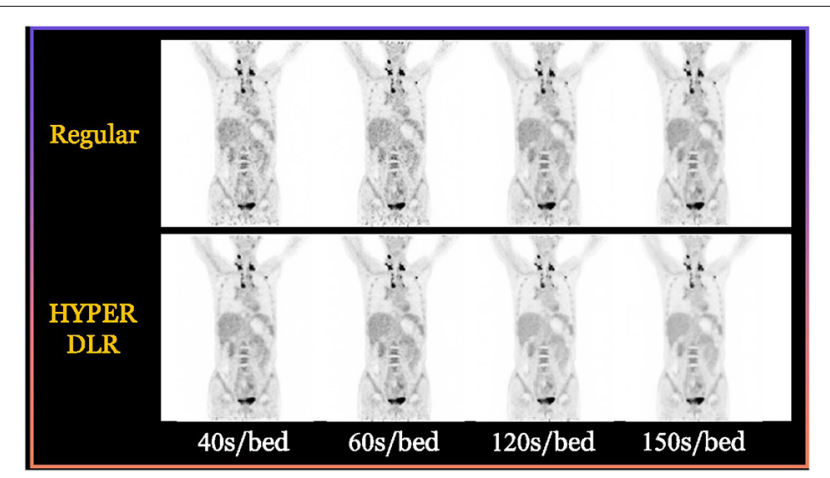

FIGURE 3 | Typical examples of the HYPER DLR PET denoising product developed by UII and UIH. The first row shows PET images obtained by using different acquisition times per bed without HYPER DLR, where the image quality degrades significantly when fast acquisition time. The second row shows the resulting images by applying the HYPER DLR technique, where obvious image quality improvement can be observed. values. This strategy has been widely adopted in many companies such as Siemens and GE. With the aid of deep learning-based cross-modality synthesis techniques, it is possible to obtain more precise synthesized CT images from the MR images with unsupervised learning techniques and therefore to produce more accurate AC operation. For instance, UII and UIH proposed an unsupervised deep learning-based technique (149) that can effectively synthesize the CT images from the MR sequences. Typical examples are shown in Figure 5.

\section{CHALLENGES AND OPPORTUNITIES}

The success of deep learning-based methods on image reconstruction for medical imaging has been extensively validated. However, the wide applications in clinical practices are not yet realized. One key limiting issue is the model interpretability. Due to the nature of DNN, the entire non-linear mapping process is a "black box," meaning that no direct physical 


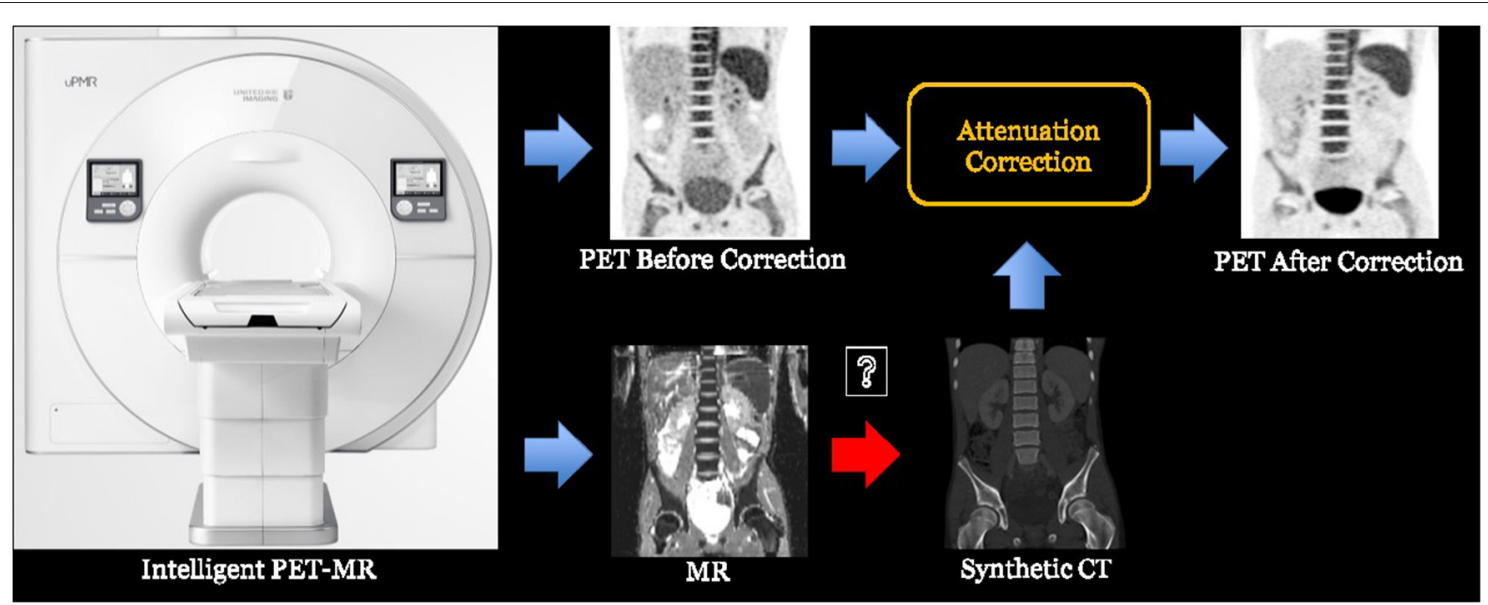

FIGURE 4 | The process of PET-MR attenuation correction, where a synthetic CT image is obtained from the MR image to help the attenuation correction of PET image.

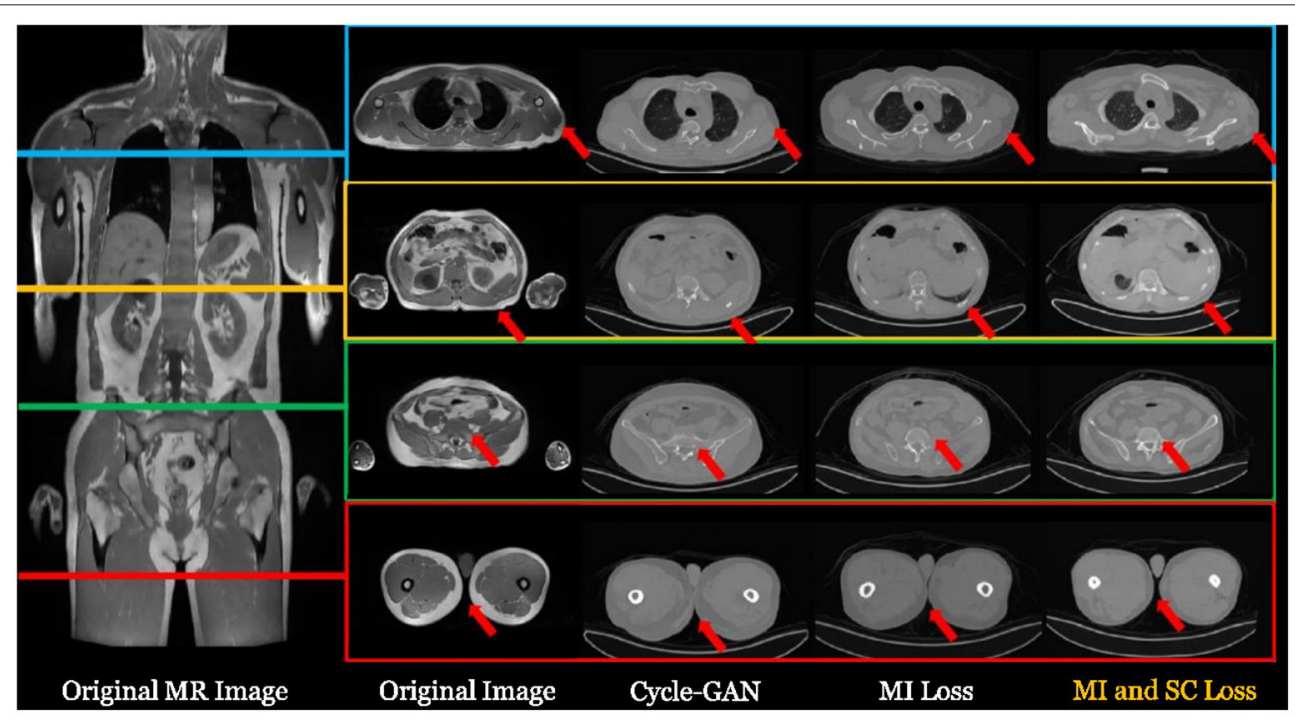

FIGURE 5 | Typical examples of synthesizing CT images from a whole body MR image with deep learning-based techniques. For more details, please refer to Ge et al. (149).

or theoretical mechanism is provided to explain how the inputs are transformed to the outputs (150). Consequently, deep learning reconstruction models find difficulties to get accepted by clinicians. Recently, enhancing model interpretability through building interpretable neural networks or utilizing various visualization techniques becomes a hot topic in deep learning-based natural image analysis (151-154). Similarly, more efforts should be devoted to building both interpretable and high-performance deep learning reconstruction models.

Another challenge is the generalization capability of deep learning-based methods. It is known that deep learning is a data-driven method, and the performance of deep learning models depends heavily on the training data $(25,26,155)$. Thus, constructing a comprehensive training dataset is critical. Different from natural images, the distributions of medical images can be quite different if different scanning protocols or scanning machines are utilized. Moreover, due to ethical issues, building large medical image datasets by collecting images from different resources is difficult. As a result, the performance of most existing deep learning models might be over-claimed, and a performance drop can be observed when applying the reported models to the data of end-users. Building robust models that can maintain performance during implementation is important to promote wide applications.

At the same time, the increasing demand for automated image analysis in the clinic to help achieve efficient and accurate imaging-based diagnosis and decision making is providing various opportunities for the introduction of deep learning-based methods. With the rapid development of computing power and optimization of deep learning models, deep learning is expected 
to play a significant role in achieving fast, portable, safe, and cheap medical imaging. For instance, the transformer (156) framework proposed in 2017 for NLP has demonstrated inspiring performance in capturing global information and has also shown great potential for applications in many image processing tasks recently. The development of the transformer also provides opportunities for the enhancement of current medical imaging models. Besides, multi-modal imaging and autonomous imaging are also promising directions for future studies.

\section{CONCLUSION}

Deep learning has presented inspiring performances in image reconstruction for different medical imaging modalities, including MRI, CT, and PET. In this review paper, we focus on the applications in MRI, CT, and PET. A detailed survey is conducted in the following aspects and sequence: the overall deep learning reconstruction workflow, the technological development of deep learning reconstruction, the clinical applications and current achievements, and a discussion of the challenges and opportunities. In summary, deep learning-based medical image reconstruction presents a great potential to promote a wide spectrum of applications in the clinic, if the remaining issues, such as interpretability and generalizability, can be properly addressed in the future.

\section{REFERENCES}

1. Shung KK, Smith M, Tsui BMW. Principles of Medical Imaging. Academic Press. (2012).

2. Doi K. Diagnostic imaging over the last 50 years: research and development in medical imaging science and technology. Phys Med Biol. (2006) 51:5-27. doi: 10.1088/0031-9155/51/13/R02

3. Westbrook C, Talbot J. MRI in Practice. John Wiley \& Sons. (2018).

4. Buzug TM. Computed Tomography. Springer handbook of medical technology. (2011):311-42. doi: 10.1007/978-3-540-74658-4_16

5. Bénard F, Romsa J, Hustinx R. Imaging gliomas with positron emission tomography and single-photon emission computed tomography. Semin Nucl Med. (2003) 33:148-62. doi: 10.1053/snuc.2003.127304

6. Edelman RR, Warach S. Magnetic resonance imaging. N Engl J Med. (1993) 328:785-91. doi: 10.1056/NEJM199303183281109

7. Hoult DI, Bhakar B. NMR signal reception: Virtual photons and coherent spontaneous emission. Concepts Magn Reson. (1997) 9:277-91. doi: 10.1002/(SICI)1099-0534(1997)9:5 < 277::AID-CMR1>3.0.CO;2-W

8. Manduca A, Yu L, Trzasko JD, Khaylova N, Kofler JM, McCollough $\mathrm{CM}$, et al. Projection space denoising with bilateral filtering and CT noise modeling for dose reduction in CT. Med Phys. (2009) 36:4911-9. doi: 10.1118/1.3232004

9. Gong Y, Shan H, Teng Y, Tu N, Liang G, Wang G, et al. Parameter-transferred Wasserstein generative adversarial network (PT-WGAN) for low-dose PET image denoising. IEEE Trans Radiat Plasma Med Sci. (2020) 5:213-23. doi: 10.1109/TRPMS.2020.3025071

10. Macovski A, Conolly S. Novel approaches to low-cost MRI. Mag Reson Med. (1993) 30:221-30. doi: 10.1002/mrm.1910300211

11. Wang S, Tan S, Gao Y, Liu Q, Ying L, Xiao T, et al. Learning joint-sparse codes for calibration-free parallel MR imaging. IEEE Trans Med Imaging. (2018) 37:251-61. doi: 10.1109/TMI.2017.2746086

12. Block KT, Uecker M, Frahm J. Undersampled radial MRI with multiple coils. Iterative image reconstruction using a total variation constraint. Mag Reson Med. (2007) 57:1086-98. doi: 10.1002/mrm.21236

\section{AUTHOR CONTRIBUTIONS}

SW: MRI review, manuscript preparation, and editing. GC: CT review, manuscript preparation, and editing. YW: PET review, manuscript preparation, and editing. SL, QW, and JS: manuscript preparation and editing. CL: manuscript preparation. DS: topic creating and manuscript editing. All authors contributed to the article and approved the submitted version.

\section{FUNDING}

This work was supported by National Natural Science Foundation of China (62131015, 62071314, 61871371, and 81830056), Science and Technology Commission of Shanghai Municipality (STCSM) (grant number 21010502600), Sichuan Science and Technology Program (2021YFG0326 and 2020YFG0079), Scientific and Technical Innovation 2030 - New Generation Artificial Intelligence Project (2020AAA0104100 and 2020AAA0104105), Key-Area Research and Development Program of Guangdong Province (2018B010109009), Key Laboratory for Magnetic Resonance and Multimodality Imaging of Guangdong Province (2020B1212060051), the Basic Research Program of Shenzhen (JCYJ20180507182400762), and Youth Innovation Promotion Association Program of the Chinese Academy of Sciences (2019351).

13. Adluru G, DiBella EVR. Reordering for improved constrained reconstruction from undersampled $\mathrm{k}$-space data. Int J Biomed Imaging. (2008) 2008:341684. doi: 10.1155/2008/341684

14. Gong K, Guan J, Kim K, Zhang X, Yang J, Seo Y, et al. Iterative PET image reconstruction using convolutional neural network representation. IEEE Trans Med Imaging. (2018) 38:675-85. doi: 10.1109/TMI.2018.2869871

15. Zhang Z, Liang X, Dong X, Xie Y, Cao G A. sparse-view CT reconstruction method based on combination of DenseNet and deconvolution. IEEE Trans Med Imaging. (2018) 37:1407-17. doi: 10.1109/TMI.2018.2823338

16. Chartrand R. Fast algorithms for nonconvex compressive sensing: MRI reconstruction from very few data. In: Proceedings of the International Symposium on Biomedical Imaging: From Nano to Macro. Boston (2009). p. 262-5. doi: 10.1109/ISBI.2009.5193034

17. Lin F, Man DB, A. hierarchical approach to deep learning and its application to tomographic reconstruction. In: Proceedings of the International Meeting on Fully Three-Dimensional Image Reconstruction in Radiology and Nuclear Medicine Philadelphia. Niskayuna (2019).

18. Fan J, Cao X, Wang Q, Yapa PT, Shen D. Adversarial learning for mono-or multi-modal registration. Med Image Anal. (2019) 58:101545. doi: 10.1016/j.media.2019.101545

19. Cao X, Yang J, Zhang J, Dong N, Kim M, Wang Q, et al. Deformable image registration based on similarity-steered $\mathrm{CNN}$ regression. In: Proceedings of the International Symposium on Medical Image Computing and Computer Assisted Intervention (MICCAI'17). Cham: Springer (2017). doi: 10.1007/978-3-319-66182-7_35

20. Luan H, Qi F, Xue Z, Chen L, Shen D. Multimodality image registration by maximization of quantitative-qualitative measure of mutual information. Pattern Recognition, (2008) 41, 285-98. doi: 10.1016/j.patcog.2007.04.002

21. Zhang J, Gao Y, Gao Y, Munsell BC, Shen D. Detecting anatomical landmarks for fast Alzheimer's disease diagnosis. IEEE Trans Med Imaging. (2016) 35:2524-33. doi: 10.1109/TMI.2016.2582386

22. Mohsen H, El-Dahshan ESA, El-Horbaty ESM, Salem ABM. Classification using deep learning neural networks for brain tumors. Future Comput Inform J. (2018) 3:68-71. doi: 10.1016/j.fcij.2017.12.001 
23. Abraham N, Khan NM. A novel focal tversky loss function with improved attention u-net for lesion segmentation. In: Proceedings of the International Symposium on Biomedical Imaging (ISBI'19) Venice. (2019). doi: 10.1109/ISBI.2019.8759329

24. Russell SJ, Norvig P. Artificial Intelligence: A Modern Approach. 3rd edn. Applied Mechanics \& Materials (2009).

25. Webb S. Deep learning for biology. Nature. (2018) 554:555-7. doi: 10.1038/d41586-018-02174-Z

26. Lecun Y, Bengio Y, Hinton G. Deep learning. Nature. (2015) 521:436-44. doi: 10.1038/nature14539

27. Shen D, Wu G, Suk HI. Deep learning in medical image analysis. Annu Rev Biomed Eng. (2017) 19:221-48. doi: 10.1146/annurev-bioeng-071516-044442

28. Murphy KP. Machine learning: a probabilistic perspective. Chance. (2014) 27:62-3. doi: 10.1080/09332480.2014.914768

29. Hastie T, Tibshirani R, Friedman J. The Elements of Statistical Learning: Data Mining. Springer Series in Statistics. (2009). doi: 10.1007/978-0-387-84858-7

30. Mnih V, Kavukcuoglu K, Silver D, Rusu AA, Veness J, Bellemare MG, et al. Human-level control through deep reinforcement learning. Nature. (2014) 518:529-33. doi: 10.1038/nature14236

31. Sutton RS, Barto AG. Reinforcement learning: an introduction. Kybernetes. (1998) 27:1093-6. doi: 10.1108/k.1998.27.9.1093.3

32. Lustig M, Donoho DL, Santos JM, Pauly JM. Compressed sensing MRI. Signal Process Mag. (2008) 25:72-82. doi: 10.1109/MSP.2007.914728

33. Liang D, Liu B, Wang J, Ying L. Accelerating SENSE using compressed sensing. Magn Reson Med. (2009) 62:1574-84. doi: 10.1002/mrm.22161

34. Qu X, Guo D, Ning B, Hou Y, Lin Y, Cai S, et al. Undersampled MRI reconstruction with patch-based directional wavelets. Magn Reson Imaging. (2012) 30:964-77. doi: 10.1016/j.mri.2012.02.019

35. Wang S, Su Z, Ling L, Peng X, Zhu S, Liang F, et al. Accelerating magnetic resonance imaging via deep learning. In: Proceedings of the International Symposium on Biomedical Imaging (ISBI'16). Prague (2016). doi: 10.1109/ISBI.2016.7493320

36. Han Y, Sunwoo L, Ye JC. k-space deep learning for accelerated MRI. IEEE Trans Med Imaging. (2020) 39:377-86. doi: 10.1109/TMI.2019.2927101

37. Zhu B, Liu JZ, Cauley SF, Rosen BR, Rosen MS. Image reconstruction by domain-transform manifold learning. Nature. (2018) 555:87-492. doi: $10.1038 /$ nature25988

38. Hammernik K, Klatzer T, Kobler E, Recht MP, Sodickson DK, Pock T, et al. Learning a variational network for reconstruction of accelerated MRI data. Magn Reson Med. (2018) 79:3055-71. doi: 10.1002/mrm.26977

39. Wang S, Xiao T, Liu Q, Zheng $H$. Deep learning for fast MR imaging: a review for learning reconstruction from incomplete k-space data. Biomed Signal Process Control. (2021) 68:102579. doi: 10.1016/j.bspc.2021.102579

40. Yang Y, Sun J, Li H, Xu Z. Deep ADMM-Net for compressive sensing MRI. NIPS. (2017) 10-18.

41. Zhang J, Ghanem B. ISTA-Net: Interpretable optimization-inspired deep network for image compressive sensing. In: Proceedings of the IEEE Conference on Computer Vision and Pattern Recognition (CVPR'18). Salt Lake (2018). p. 1828-37. doi: 10.1109/CVPR.2018.00196

42. Adler J, Öktem O. Solving ill-posed inverse problems using iterative deep neural networks. Inverse Probl. (2017) 33:124007. doi: 10.1088/1361-6420/aa9581

43. Wang S, Cheng $\mathrm{H}$, Ying L, Xiao $\mathrm{T}$, Ke Z, Zheng $\mathrm{H}$, et al. Deep complex MRI: exploiting deep residual network for fast parallel MR imaging with complex convolution. Magn Reson Imaging. (2020) 68:136-47. doi: 10.1016/j.mri.2020.02.002

44. Quan TM, Nguyen-Duc T, Jeong WK. Compressed sensing MRI reconstruction using a generative adversarial network with a cyclic loss. IEEE Trans Med Imaging. (2018) 37:1488-97. doi: 10.1109/TMI.2018.2820120

45. Feldkamp LA, Davis LC, Kress JW. Practical cone-beam algorithm. J Opt Soc Am. (1984) 1:612-9. doi: 10.1364/JOSAA.1.000612

46. Gordon R, Bender R, Herman GT. Algebraic reconstruction techniques (ART) for three-dimensional electron microscopy and X-ray photography. J Theor Biol. (1970) 29:477-81. doi: 10.1016/0022-5193(70)90109-8

47. Andersen $\mathrm{AH}$, Kak AC. Simultaneous algebraic reconstruction technique (SART): a superior implementation of the ART algorithm. Ultrason Imaging. (1984) 6:81-94. doi: 10.1177/016173468400600107
48. Erdogan H, Fessler JA. Ordered subsets algorithms for transmission tomography. Phys Med Biol. (1999) 44:2835-51. doi: 10.1088/0031-9155/44/11/311

49. Lange $\mathrm{K}$, Carson R. EM reconstruction algorithms for emission and transmission tomography. J Comput Assist Tomogr. (1984) 8:306-16. doi: 10.1097/00004728-198404000-00002

50. Thibault JB, Sauer KD, Bouman CA, Hsieh J. A three-dimensional statistical approach to improved image quality for multislice helical CT. Med Phys. (2007) 34:4526-44. doi: 10.1118/1.2789499

51. Zhang Z, Yu S, Qin W, Liang X, Xie Y, Cao G. Self-supervised CT super-resolution with hybrid model. Comput Biol Med. (2021) 138:104775. doi: 10.1016/j.compbiomed.2021.104775

52. Yang G, Yu S, Dong H, Slabaugh G, Dragotti PL, Ye X, et al. DAGAN: deep De-aliasing generative adversarial networks for fast compressed sensing MRI reconstruction. IEEE Trans Med Imaging. (2018) 37:1310-21. doi: 10.1109/TMI.2017.2785879

53. Kang E, Min J, Ye JC. A deep convolutional neural network using directional wavelets for low-dose X-ray CT reconstruction. Med Phys. (2016) 44:e36075. doi: $10.1002 / \mathrm{mp} .12344$

54. Chen H, Zhang Y, Kalra NK, Lin F, Chen Y, Liao P, et al. Low-dose CT with a residual encoder-decoder convolutional neural network. IEEE Trans Med Imaging. (2017) 36:2524-35. doi: 10.1109/TMI.2017.2715284

55. Zhang C, Xing Y. CT artifact reduction via U-net CNN. In: Proceedings of the Medical Imaging 2018: Image Processing (2018). doi: 10.1117/12.2293903

56. Han Y, Ye JC. Framing U-Net via deep convolutional framelets: application to sparse-view CT. IEEE Trans Med Imaging. (2018) 37:1418-29. doi: 10.1109/TMI.2018.2823768

57. Park J, Hwang D, YunKim K, Kang S, Kim YK, Lee JS. Computed tomography super-resolution using deep convolutional neural network. Phys Med Biol. (2018) 63:145011. doi: 10.1088/1361-6560/aacdd4

58. Samuel Matej S, Daube-Witherspoon ME, Karp JS. Analytic TOF PET reconstruction algorithm within DIRECT data partitioning framework. Phys Med Biol. (2016) 61:3365-86. doi: 10.1088/0031-9155/61/9/3365

59. Defrise M, Kinahan P. Data Acquisition and Image Reconstruction for $3 d 33 d$ Pet. Springer (1998). p. 11-53. doi: 10.1007/978-94-017-3475-2_2

60. Shepp LA, Vardi Y. Maximum likelihood reconstruction for emission tomography. IEEE Trans Med Imaging. (1982) 1:113-22. doi: 10.1109/TMI.1982.4307558

61. Hudson HM, Larkin RS. Accelerated image reconstruction using ordered subsets of projection data. IEEE Trans Med Imaging. (1994) 13:601-9. doi: $10.1109 / 42.363108$

62. Kang J, Gao Y, Shi F, Lalush DS, Lin W, Shen D. Prediction of standard-dose brain PET image by using MRI and low-dose brain [18F]FDG PET images. Med Phys. (2015) 42:5301-9. doi: 10.1118/1.4928400

63. Wang Y, Zhang P, An L, Ma G, Kang J, Shi F, et al. Predicting standarddose PET image from low-dose PET and multimodal MR images using mapping-based sparse representation. Phys Med Biol. (2016) 61:791-812. doi: 10.1088/0031-9155/61/2/791

64. An L, Zhang P, Adeli E, Wang Y, Ma G, Shi F, et al. Multi-level canonical correlation analysis for standard-dose PET image estimation. IEEE Trans Image Process. (2016) 25:3303-15. doi: 10.1109/TIP.2016.2567072

65. Xiang L, Qiao Y, Nie D, An L, Lin W, Wang Q, et al. Deep autocontext convolutional neural networks for standard-dose PET image estimation from low-dose PET/MRI. Neurocomputing. (2017) 267:406-16. doi: 10.1016/j.neucom.2017.06.048

66. Xu J, Gong E, Pauly J, Zaharchuk G. 200x Low-dose PET reconstruction using deep learning. (2017). arXiv:1712.04119.

67. Wang Y, Zhou L, Yu B, Wang L, Zu C, Lalush DS, et al. 3D autocontext-based locality adaptive multi-modality GANs for PET synthesis. IEEE Trans Med Imaging. (2019) 38:1328-39. doi: 10.1109/TMI.2018.28 84053

68. Kim K, Wu D, Gong K, Dutta J, Kim JH, Son Y, et al. Penalized PET reconstruction using deep learning prior and local linear fitting. IEEE Trans Med Imaging. (2018) 37:1478-87. doi: 10.1109/TMI.2018.2832613

69. Gong K, Catana C, Qi J, Li Q. PET image reconstruction using deep image prior. IEEE Trans Med Imaging. (2019) 38:1655-65. doi: 10.1109/TMI.2018.2888491 
70. Feng Q, Liu H. Rethinking PET image reconstruction: ultra-lowdose, sinogram and deep learning. In: Proceedings of the International Conference on Medical Image Computing and Computer Assisted Intervention (MICCAI'20) (2020). p. 783-92. doi: 10.1007/978-3-030-59728-3_76

71. Liu Z, Chen H, Liu H. Deep learning based framework for direct reconstruction of PET images. In: Proceedings of the International Conference on Medical Image Computing and Computer Assisted Intervention (MICCAI'19). Springer (2019). p. 48-56. doi: 10.1007/978-3-030-32248-9_6

72. Zhou L, Schaefferkoetter JD, Tham IWK, Gang H, Yan J. Supervised learning with CycleGAN for low-dose FDG PET image denoising. Med Image Anal. (2020) 65:101770. doi: 10.1016/j.media.2020.101770

73. Huang Q, Xian Y, Yang D, Qu H, Yi J, Wu P, et al. Dynamic MRI reconstruction with end-to-end motion-guided network. Med Image Anal. (2020) 68:1010901. doi: 10.1016/j.media.2020.101901

74. Wang S, Ke Z, Cheng H, Jia S, Ying L, Zheng H, et al. DIMENSION: dynamic MR imaging with both $\mathrm{k}$-space and spatial prior knowledge obtained via multi-supervised network training. NMR Biomed. (2019) 1-16. doi: $10.1002 / \mathrm{nbm} .4131$

75. Aggarwal HK, Mani MP, Jacob M. MoDL: Model-based deep learning architecture for inverse problems. IEEE Trans Med Imaging. (2019) 38:394405. doi: 10.1109/TMI.2018.2865356

76. Chen Y, Xiao T, Li C, Liu Q, Wang S. Model-based convolutional de-aliasing network learning for parallel MR imaging. In: Proceedings of the International Conference on Medical Image Computing and Computer Assisted Intervention (MICCAI'19). Springer (2019). p. 30-8. doi: 10.1007/978-3-030-32248-9_4

77. Yaman B, Hosseini SAH, Moeller S, Ellermann J, Ugurbil K, Akçakaya M. Self-supervised physics-based deep learning MRI reconstruction without fully-sampled data. In: Proceedings of the International Symposium on Biomedical Imaging (ISBI'20). Iowa City (2020). doi: 10.1109/ISBI45749.2020.9098514

78. Hu Y, Xu Y, Tian Q, Chen F, Shi X, Moran CJ, et al. RUN-UP: accelerated multishot diffusion-weighted MRI reconstruction using an unrolled network with U-net as priors. Magn Reson Med. (2020) 85:709-20. doi: $10.1002 / \mathrm{mrm} .28446$

79. Kang E, Cahng W, Yoo J, Ye JC. Deep convolutional framelet denosing for low-dose CT via wavelet residual network. IEEE Trans Med Imaging. (2018) 37:1358-69. doi: 10.1109/TMI.2018.2823756

80. Hwan K, McCann MT, Froustey E, Unser M. Deep convolutional neural network for inverse problems in imaging. IEEE Trans Image Process. (2017) 26:4509-22. doi: 10.1109/TIP.2017.2713099

81. Wolterink JM, Leiner T, Viergever MA, Išgum I. Generative adversarial networks for noise reduction in low-dose CT. IEEE Trans Med Imaging. (2017) 36:2536-45. doi: 10.1109/TMI.2017.2708987

82. Yang Q, Yan P, Zhang Y, Yu H, Shi Y, Mou X, et al. Low-dose CT image denoising using a generative adversarial network with wasserstein distance and perceptual loss. IEEE Trans Med Imaging. (2018) 37:1348-57. doi: 10.1109/TMI.2018.2827462

83. You C, Li G, Zhang Y, Zhang X, Shan H, Li M, et al. CT superresolution GAN constrained by the identical, residual, and cycle learning ensemble (GAN-CIRCLE). IEEE Trans Med Imaging. (2020) 39:188-203. doi: 10.1109/TMI.2019.2922960

84. Gupta H, Jin KH, Nguyen HQ, McCann MT, Unser M. CNN-based projected gradient descent for consistent CT image reconstruction. IEEE Trans Med Imaging. (2018) 37:1440-53. doi: 10.1109/TMI.2018.2832656

85. Xiang L, Wang L, Gong E, Zaharchuk G, Zhang T. Noise-aware standarddose PET reconstruction using general and adaptive robust loss. In: Proceedings of the International Conference on Machine Learning in Medical Imaging (MLMI'20). (2020). p. 654-62. doi: 10.1007/978-3-030-59861-7_66

86. Bevilacqua A, Bollini D, Campanini R, Lanconelli N, Galli M. A new approach to image reconstruction in positron emission tomography using artificial neural networks. Int J Mod Phys C. (1998) 9:71-85. doi: 10.1142/S0129183198000078

87. Yang B, Ying L, Tang J. Artificial neural network enhanced Bayesian PET image reconstruction. IEEE Trans Med Imaging. (2018) 37:1297-309. doi: 10.1109/TMI.2018.2803681

88. Spuhler K, Serrano-Sosala M, Cattell R, DeLorenzo C, Huang C. Full-count PET recovery from low-countimage using a dilated convolutional neural network. Med Phys. (2020) 47:4928-38. doi: 10.1002/mp.14402
89. Whiteley W, Luk WK, Gregor J, DirectPET: full-size neural network PET reconstruction from sinogram data. J Med Imag. (2020) 7: 032503. doi: 10.1117/1.JMI.7.3.032503

90. Xie Z, Li T, Zhang X, Qi W, Asma E, Qi J. Anatomically aided PET image reconstruction using deep neural networks. Med Phys. (2021) 48:5244-58. doi: $10.1002 / \mathrm{mp} .15051$

91. Sandino CM, Lai P, Vasanawala SS, Cheng JY. Accelerating cardiac cine MRI using a deep learning-based ESPIRiT reconstruction. Magn Reson Med. (2021) 85:152-67. doi: 10.1002/mrm.28420

92. Lee D, Yoo J, Ye JC. Deep residual learning for compressed sensing MRI. In: Proceedings of the International Symposium on Biomedical Imaging (ISBI'17). Melbourne (2017). p. 15-8. doi: 10.1109/ISBI.2017.7950457

93. Ke Z, Cheng J, Ying L, Zheng H, Zhu Y, Liang D. An unsupervised deep learning method for multi-coil cine MRI. Phys Med Biol. (2020) 65:235041. doi: 10.1088/1361-6560/abaffa

94. Liu X, Zhang M, Liu Q, Xiao T, Zheng H, Ying L, et al. Multi-contrast $M R$ reconstruction with enhanced denoising autoencoder prior learning. In: Proceedings of the International Symposium on Biomedical Imaging (ISBI'20). Iowa (2020). p. 1432-6. doi: 10.1109/ISBI45749.2020.9098334

95. Mills AF, Sakai O, Anderson SW, Jara H. Principles of quantitative MR imaging with illustrated review of applicable modular pulse diagrams. Radiographics. (2017) 37:2083-105. doi: 10.1148/rg.2017160099

96. Kwee TC, Kwee RM. Chest CT in COVID-19: What the radiologist needs to know. Radiographics. (2020) 40:1848-65. doi: 10.1148/rg.2020200159

97. Vayá MDLI, Saborit JM, Montell JA, Pertusa A, Bustos A, Cazorla M, et al. BIMCV COVID-19+: a large annotated dataset of RX and CT images from COVID-19 patients. (2020). arXiv:2006.01174.

98. Wang H, Xu Y, Zhao Y, Zhao Y. A novel static PET image reconstruction method. In: Proceedings of the Chinese Automation Congress (CAC'17). Jinan (2017). p. 4537-41. doi: 10.1109/CAC.2017.8243580

99. Serrano-Sosa M, Spuhler K, DeLorenzo C, Huang C. PET image denoising using structural MRI with a novel dilated convolutional neural network. $J$ Nucl Med. (2020) 61:434.

100. Ladefoged CN, Hasbak P, Hornnes C, Højgaard L, Andersen FL. Lowdose PET image noise reduction using deep learning: application to cardiac viability FDG imaging in patients with ischemic heart disease. Phys Med Biol. (2021) 66:054003. doi: 10.1088/1361-6560/abe225

101. Novosad P, Reader AJ. MR-guided dynamic PET reconstruction with the kernel method and spectral temporal basis functions. Phys Med Biol. (2016) 61:4624-45. doi: 10.1088/0031-9155/61/12/4624

102. Cui J, Liu X, Wang Y, Liu H. Deep reconstruction model for dynamic PET images. PLoS ONE. (2017) 12:e0184667. doi: 10.1371/journal.pone. 0184667

103. Gong K, Guan J, Liu CC, Qi J. PET image denoising using a deep neural network through fine tuning. IEEE Trans Radiat Plasma Med Sci. (2018) 3:153-61. doi: 10.1109/TRPMS.2018.2877644

104. Sun H, Peng L, Zhang H, He Y, Cao S, Lu L. Dynamic PET image denoising using deep image prior combined with regularization by denoising. IEEE Access. (2021) 9:52378-92. doi: 10.1109/ACCESS.2021.3069236

105. Ouyang J, Chen KT, Gong E, Pauly J, Zaharchuk G. Ultra-low-dose PET reconstruction using generative adversarial network with feature matching and task-specific perceptual loss. Med Phys. (2019) 46:3555-64. doi: $10.1002 / \mathrm{mp} .13626$

106. Xu J, Liu H. Three-dimensional convolutional neural networks for simultaneous dual-tracer PET imaging. Phys Med Biol. (2019) 64:185016. doi: 10.1088/1361-6560/ab3103

107. Xu J, Liu H. Hybrid-loss guided 3D CNN for dynamic dual-tracer PET reconstruction. In: Proceedings of the International Symposium on Biomedical Imaging (ISBI'19). Venice (2019).

108. Ellis S, Mallia A, McGinnity CJ, Cook GJR, reader AJ. Guided image reconstruction for multi-tracer PET. In: Proceedings of the Nuclear Science Symposium and Medical Imaging Conference (NSS/MIC'17). Atlanta (2017). doi: 10.1109/NSSMIC.2017.8533140

109. Hong X, Zan Y, Weng F, Tao W, Peng Q, Huang Q. Enhancing the image quality via transferred deep residual learning of coarse PET sinograms. IEEE Trans Med Imaging. (2018) 37:2322-32. doi: 10.1109/TMI.2018.2830381

110. Häggström I, Schmidtlein C R, Campanella G, Fuchs TJ. DeepPET: a deep encoder-decoder network for directly solving the PET image 
reconstruction inverse problem. Med Image Anal. (2019) 54:253-62. doi: 10.1016/j.media.2019.03.013

111. Kwon K, Kim D, Park H. A parallel MR imaging method using multilayer perceptron. Med Phys. (2017) 44:6209-24. doi: 10.1002/mp.12600

112. Ronneberger O, Fischer P, Brox T. U-Net: Convolutional networks for biomedical image segmentation. In: Proceedings of the International Conference on Medical Image Computing and Computer Assisted Intervention (MICCAI'15). Springer (2015). p. 234-41. doi: 10.1007/978-3-319-24574-4_28

113. Falk T, Mai D, Bensch R, Çiçek Ö, Abdulkadir A, Marrakchi Y, et al. U-Net: deep learning for cell counting, detection, and morphometry. Nat Methods. (2019) 16:67-70. doi: 10.1038/s41592-018-0261-2

114. Lee D, Yoo J, Tak S, Ye JC. Deep residual learning for accelerated MRI using magnitude and phase networks. IEEE Trans Biomed Eng. (2018) 65:1985-95. doi: 10.1109/TBME.2018.2821699

115. Han Y, Yoo J, Kim HH, Shin HJ, Sung K, Ye JC. Deep learning with domain adaptation for accelerated projection-reconstruction MR. Magn Reson Med. (2018) 80:1189-205. doi: 10.1002/mrm.27106

116. Duan C, Deng H, Xiao S, Xie J, Li H, Sun X, et al. Fast and accurate reconstruction of human lung gas MRI with deep learning. Magn Reson Med. (2019) 82:2273-85. doi: 10.1002/mrm.27889

117. Hyun CM, Kim HP, Lee SM, Lee S, Seo JK. Deep learning for undersampled MRI reconstruction. Phys Med Biol. (2018) 63:135007. doi: 10.1088/1361-6560/aac71a

118. Kofler A, Haltmeier M, Kolbitsch C, Kachelrieß M, Dewey M. A UNets cascade for sparse view computed tomography. In: Proceedings of the Machine Learning for Medical Image Reconstruction (MLMIR'18). Springer (2018). p. 91-9. doi: 10.1007/978-3-030-00129-2_11

119. Liu Y, Zhang Y. Low-dose CT restoration via stacked sparse denoising autoencoders. Neurocomputing. (2018) 284:80-9. doi: 10.1016/j.neucom.2018.01.015

120. Schaefferkoetter J, Yan, J, Ortega C, Sertic A, Lechtman E, Eshet Y, et al. Convolutional neural networks for improving image quality with noisy PET data. EJNMMI Res. (2020) 10:105. doi: 10.1186/s13550-020-00695-1

121. Sikka A, Peri SV, Bathula DR. MRI to FDG-PET: cross-modal synthesis using 3D U-Net for multi-modal Alzheimer's classification. In: Proceedings of the International Conference on Simulation and Synthesis in Medical Imaging (SASHIMI'18). Springer (2018). doi: 10.1007/978-3-030-00536-8_9

122. Cui J, Gong K, Guo N, Wu C, Meng X, Kim K, et al. PET image denoising using unsupervised deep learning. Eur J Nucl Med Mol Imaging. (2019) 46:2780-9. doi: 10.1007/s00259-019-04468-4

123. He K, Zhang X, Ren S, Sun J. Deep residual learning for image recognition. In: Proceedings of the International Conference on Computer Vision and Pattern Recognition (CVPR'16). Las Vegas (2016). p. 770-8.

124. Shi J, Liu Q, Wang C, Zhang Q, Ying S, Xu H. Super-resolution reconstruction of MR image with a novel residual learning network algorithm. Phys Med Biol. (2018) 63:085011. doi: 10.1088/1361-6560/aab9e9

125. Shi J, Li Z, Ying S, Wang C, Zhang Q, Yan P, et al. image super-resolution via wide residual networks with fixed skip connection. IEEE J Biomed Health Inform. (2019) 23:1129-40. doi: 10.1109/JBHI.2018.2843819

126. Li X, Cao T, Tong Y, Ma X, Niu Z, Guo H. Deep residual network for highly accelerated fMRI reconstruction using variable density spiral trajectory. Neurocomputing. (2020) 398:338-46. doi: 10.1016/j.neucom.2019. 02.070

127. Du J, He Z, Wang L, Gholipour A, Zhou Z, Chen D, et al. Superresolution reconstruction of single anisotropic $3 \mathrm{D}$ MR images using residual convolutional neural network. Neurocomputing. (2020) 392:209-20. doi: 10.1016/j.neucom.2018.10.102

128. Chen KT, Gong E, de Carvalho Macruz FB, Xu J, Boumis A, Khalighi $\mathrm{M}$, et al. Ultra-low-dose $18 \mathrm{~F}$-florbetaben amyloid PET imaging using deep learning with multi-contrast MRI inputs. Radiology. (2019) 290:649-56. doi: 10.1148/radiol.2018180940

129. Dong X, Vekhande S, Cao G. Sinogram interpolation for sparse-view microCT with deep learning neural network. In: Proceedings of the Medical Imaging 2019: Physics of Medical Imaging (2019).

130. Goodfellow IJ, Pouget-Abadie J, Mirza M, Xu B, Warde-Farley D, Ozair, S, et al. Generative adversarial nets. Adv Neural Inform Processing Syst. (2014) 2672-80.
131. Mardani M, Gong E, Cheng JY, Vasanawala SS, Zaharchuk G, Xing L, et al. Deep generative adversarial neural networks for compressive sensing MRI. IEEE Trans Med Imaging. (2019) 38:167-79. doi: 10.1109/TMI.2018.2858752

132. Simonyan K, Zisserman A. Very deep convolutional networks for large-scale image recognition. (2014). arXiv:1409.1556.

133. Wang Y, Yu B, Wang L, Zu C, Lalush DS, Lin W, et al. 3D conditional generative adversarial networks for high-quality PET image estimation at low dose. Neuroimage. (2018) 174:550-62. doi: 10.1016/j.neuroimage.2018.03.045

134. Ben-Cohen A, Klang E, Raskin SP, Soffer S, Ben-Haim S, Kone E. et al. Cross-modality synthesis from CT to PET using FCN and GAN networks for improved automated lesion detection. Eng Appl Artif Intell. (2019) 78:186-94. doi: 10.1016/j.engappai.2018.11.013

135. Yaakub SN, McGinnity CJ, Clough JR, Kerfoot E, Girard N, Guedj E, et al. Pseudo-normal PET synthesis with generative adversarial networks for localising hypometabolism in epilepsies. In: Proceedings of the International Workshop on Simulation and Synthesis in Medical Imaging. (2019). doi: 10.1007/978-3-030-32778-1_5

136. Kaplan S, Zhu Y-M. Full-dose PET image estimation from low-dose PET image using deep learning: a pilot study. J Digit Imaging. (2019) 32:773-8. doi: 10.1007/s10278-018-0150-3

137. Yang L, Dong $\mathrm{X}$, Wang $\mathrm{T}$, Higgins $\mathrm{K}$, Liu $\mathrm{T}$, Curran WJ, et al. Whole-body PET estimation from low count statistics using cycleconsistent generative adversarial networks. Phys Med Biol. (2019) 64:215017. doi: 10.1088/1361-6560/ab4891

138. Schlemper J, Caballero J, Hajnal JV, Price AN, Rueckert D, A. deep cascade of convolutional neural networks for dynamic MR image reconstruction. IEEE Trans Med Imaging. (2018) 37:491-503. doi: 10.1109/TMI.2017.2760978

139. Qin C, Schlemper J, Caballero J, Price AN, Hajnal JV, Rueckert D. Convolutional recurrent neural networks for dynamic MR image reconstruction. IEEE Trans Med Imaging. (2019) 38:280-90. doi: 10.1109/TMI.2018.2863670

140. Ran M, Xia W, Huang Y, Lu Z, Bao P, Liu Y, et al. MD-ReconNet: a parallel dual-domain convolutional neural network for compressed sensing MRI. IEEE Trans Radiat Plasma Med Sci. (2020) 5:120-35. doi: 10.1109/TRPMS.2020.2991877

141. El-Rewaidy H, Neisius U, Mancio J, Kucukseymen S, Rodriguez J, Paskavitz A, et al. Deep complex convolutional network for fast reconstruction of 3D late gadolinium enhancement cardiac MRI. NMR Biomed. (2020) 33:e4312. doi: $10.1002 / \mathrm{nbm} .4312$

142. Cole E, Cheng J, Pauly J, Vasanawala S. Analysis of deep complex-valued convolutional neural networks for MRI reconstruction and phase-focused applications. Magn Reson Med. (2021) 86:1093-109. doi: 10.1002/mrm.28733

143. Shen C, Gonzalez Y, Chen L, Jiang SB, Jia X. Intelligent parameter tuning in optimization-based iterative CT reconstruction via deep reinforcement learning. IEEE Trans Med Imaging. (2018) 37:1430-9. doi: 10.1109/TMI.2018.2823679

144. Du Q, Qiang Y, Yang W, Wang Y, Muhhammad BZ. DRGAN: a deep residual generative adversarial network for PET image reconstruction. IET Image Processing. (2020) 14:1690-700. doi: 10.1049/iet-ipr.2019.1107

145. Huang Q, Yang D, Wu P, Qu H, Yi J, Metaxas D, et al. reconstruction via cascaded channel-wise attention network. In: Proceedings of the International Symposium on Biomedical Imaging (ISBI'19). Venice (2019). doi: 10.1109/ISBI.2019.8759423

146. Zhang T, Jackson1 LH, Uus A, Clough JR, Story L, Rutherford MA, et al. Self-supervised recurrent neural network for $4 \mathrm{D}$ abdominal and In-utero $\mathrm{MR}$ imaging. In: International Workshop on Machine Learning for Medical Image Reconstruction. Springer (2019). doi: 10.1007/978-3-030-33843-5_2

147. Shan H, Padole A, Homayounieh F, Kruger U, Khera RD, Nitiwarangkul C, et al. Competitive performance of a modularized deep neural network compared to commercial algorithms for low-dose CT image reconstruction. Nat Mach Intell. (2019) 1:269-76. doi: 10.1038/s42256-019-0057-9

148. Xiang L, Wang Q, Nie D, Zhang L, Jin X, Qiao Y, et al. Deep embedding convolutional neural network for synthesizing CT image from T1-Weighted MR image. Med Image Anal. (2018) 47:31-44. doi: 10.1016/j.media.2018.03.011

149. Ge Y, Wei D, Xue Z, Wang Q, Zhou X, Zhan Y. Unpaired Mr to CT synthesis with explicit structural constrained adversarial learning. In: Proceedings of 
the International Symposium on Biomedical Imaging (ISBI'19). Venice (2019). p. 1096-99.

150. Koh PW, Liang P. Understanding black-box predictions via influence functions. In: Proceedings of the International Conference on Machine Learning (PMLR'17). Sydney (2017). p. 2976-87.

151. Zhang Q, Wu Y, Zhu S-C. Interpretable convolutional neural networks. In: Proceedings of the International Conference on Computer Vision and Pattern Recognition (CVPR'18). Salt Lake (2018). p. 8827-36. doi: 10.1109/CVPR.2018.00920

152. Pope PE, Kolouri S, Rostami M, Martin CE, Hoffmann H. Explainability methods for graph convolutional neural networks. In: Proceedings of the International Conference on Computer Vision and Pattern Recognition (CVPR'19). Long Beach (2019). p. 10772-81. doi: 10.1109/CVPR.2019.01103

153. Zhou B, Khosla A, Lapedriza A, Oliva A, Torralba A. Learning deep features for discriminative localization. In: Proceedings of the International Conference on Computer Vision and Pattern Recognition (CVPR'16). Las Vegas (2016). p. 2921-9. doi: 10.1109/CVPR.2016.319

154. Selvaraju RR, Cogswell M, Das A, Vedantam R, Parikh D, Batra D. Grad-CAM: visual explanations from deep networks via gradient-based localization. In: Proceedings of the International Conference on Computer Vision and Pattern Recognition (CVPR'17). Springer (2017). p. 618-26. doi: 10.1109/ICCV.2017.74

155. Towards trustable machine learning. Nat Biomed Eng. (2018) 2:709-10. doi: $10.1038 / \mathrm{s} 41551-018-0315-\mathrm{x}$
156. Vaswani A, Shazeer N, Parmar N, Uszkoreit J, Jones L, Gomez AN, et al. Attention is all you need. (2017). arXiv:1706.03762.

Conflict of Interest: SL and DS are employed by Shanghai United Imaging Intelligence Co., Ltd.

The remaining authors declare that the research was conducted in the absence of any commercial or financial relationships that could be construed as a potential conflict of interest.

Publisher's Note: All claims expressed in this article are solely those of the authors and do not necessarily represent those of their affiliated organizations, or those of the publisher, the editors and the reviewers. Any product that may be evaluated in this article, or claim that may be made by its manufacturer, is not guaranteed or endorsed by the publisher.

Copyright (๑) 2021 Wang, Cao, Wang, Liao, Wang, Shi, Li and Shen. This is an open-access article distributed under the terms of the Creative Commons Attribution License (CC BY). The use, distribution or reproduction in other forums is permitted, provided the original author(s) and the copyright owner(s) are credited and that the original publication in this journal is cited, in accordance with accepted academic practice. No use, distribution or reproduction is permitted which does not comply with these terms. 